\title{
Plasma membrane phosphatidylinositol-4-phosphate is not necessary for Candida albicans viability, yet is key for cell wall integrity and systemic infection
}

Rocio Garcia-Rodas ${ }^{1}$, Hayet Labbaoui ${ }^{1}$, François Orange ${ }^{2}$, Norma Solis ${ }^{3}$, Oscar

Zaragoza $^{4}$, Scott Filler ${ }^{3,5}$, Martine Bassilana ${ }^{1}$ and Robert A. Arkowitz ${ }^{1 *}$

${ }^{1}$ Université Côte d'Azur, CNRS, INSERM, Institute of Biology Valrose (iBV), Parc Valrose, Nice, FRANCE.

${ }^{2}$ Université Côte d'Azur, CCMA, Parc Valrose, Nice, FRANCE.

${ }^{3}$ Institute for Infection and Immunity, Lundquist Institute for Biomedical Innovation at Harbor-UCLA Medical Center, Torrance, CA, USA.

${ }^{4}$ Mycology Reference Laboratory, National Centre for Microbiology, Health Institute Carlos III, Majadahonda, Madrid, Spain.

${ }^{5}$ David Geffen School of Medicine at UCLA, Los Angeles, CA, USA.

*Correspondence: arkowitz@unice.fr

Running title: Role of plasma membrane PI4P in C. albicans 


\section{Abstract}

Phosphatidylinositol phosphates are key phospholipids with a range of regulatory roles, including membrane trafficking and cell polarity. Phosphatidylinositol-4-phosphate $[\mathrm{PI}(4) \mathrm{P}]$ at the Golgi is required for the budding to filamentous growth transition in the human pathogenic fungus Candida albicans, however the role of plasma membrane $\mathrm{PI}(4) \mathrm{P}$ is unclear. We have investigated the importance of this phospholipid in C. albicans growth, stress response, and virulence by generating mutant strains with decreased levels of plasma membrane PI(4)P, via deletion of components of the PI-4-kinase complex, i.e. Efr3, Ypp1 and Stt4. The amount of plasma membrane PI(4)P in the efr $3 \Delta / \Delta$ and $y p p 1 \Delta / \Delta$ mutant was $\sim 60 \%$ and $\sim 40 \%$ of the wild-type strain, respectively, whereas it was nearly undetectable in the stt $4 \Delta / \Delta$ mutant. All three mutants had reduced plasma membrane phosphatidylserine (PS). Although these mutants had normal yeast phase growth, they were defective in filamentous growth, exhibited defects in cell wall integrity and had an increased exposure of cell wall $\beta(1,3)$-glucan, yet they induced a range of hyphal specific genes. In a mouse model of hematogenously disseminated candidiasis, fungal plasma membrane PI(4)P levels directly correlated with virulence; the efr $3 \Delta / \Delta$ had wild-type virulence, the $\operatorname{ypp} 1 \Delta / \Delta$ mutant had attenuated virulence and the $\operatorname{stt} 4 \Delta / \Delta$ mutant caused no lethality. In the mouse model of orpharyngeal candidiasis, only the $y p p 1 \Delta / \Delta$ mutant had reduced virulence, indicating that plasma membrane $\mathrm{PI}(4) \mathrm{P}$ is less important for proliferation in the oropharynx. Collectively, these results demonstrate that plasma membrane PI(4)P levels play a central role in filamentation, cell wall integrity and virulence in C. albicans. 


\section{Importance}

While the PI-4-kinases Pik1 and Stt4 both produce PI(4)P, the former generates PI(4)P at the Golgi and the latter at the plasma membrane and these two pools are functionally distinct. To address the importance of plasma membrane PI(4)P in Candida albicans, we have generated deletion mutants of the three putative plasma membrane PI-4-kinase complex components and quantified the levels of plasma membrane PI(4)P in each of these strains. Our work reveals that this phosphatidylinositol phosphate is specifically critical for the yeast-to-hyphal transition, cell wall integrity and virulence in a mouse systemic infection model. The significance of this work is in identifying a plasma membrane phospholipid that has an infection specific role, which is attributed to the loss of plasma membrane $\mathrm{PI}(4) \mathrm{P}$ resulting in $\beta(1,3)$-glucan unmasking. 


\section{Introduction}

Phosphatidylinositol phosphates are minor components of cellular membranes that play an essential role during polarized growth. In particular, phosphatidylinositol-4-phosphate $[\mathrm{PI}(4) \mathrm{P}]$ is found predominantly at the Golgi and plasma membranes, generated from the precursor PI which is synthesized at the cytosolic face of the ER (1). Type III PI4-kinases are found in virtually all eukaryotes, with Pik1 in fungi homologous to mammalian type III $\beta$ PI4-kinases and Stt4 to mammalian type III $\alpha$ PI4-kinases $(2,3)$. While all fungi appear to have Stt4 orthologs, Pik1 orthologs are absent in some of them, including Aspergillus nidulans and Cryptococcus neoformans $(4,5)$ and it has been suggested that Stt4 can carry out Pik1 essential function in these fungi. Plasma membrane PI(4)P generated by Stt4, has been implicated in the control of membrane trafficking, lipid exchange, cell signaling, cytoskeleton organization and cytokinesis. Stt4 type III $\alpha$ PI4kinases are essential for viability in most fungi including Saccharomyces cerevisiae and Schizosaccharomyces pombe (6-9), however stt4 mutants can be rescued by osmoremediation $(6,9)$.

Candida albicans is a major human fungal opportunistic pathogen that grows in both yeast and filamentous forms. The morphological transition between yeast and filamentous growth is important for its virulence and can be triggered by a range of stimuli (10-12). Using mutants in which the levels of phosphatidylinositol phosphates can be reduced revealed that Golgi $\mathrm{PI}(4) \mathrm{P}$ and plasma membrane $\mathrm{PI}(4,5) \mathrm{P}_{2}$ are critical for this transition $(13,14)$. Mutants with reduced levels of the plasma membrane PI-4-kinase Stt4, can nonetheless form germ tubes and grow invasively (14), raising the question as to whether this pool of PI(4)P is critical for the yeast-to-hyphal transition. In C. albicans (13), as in S. cerevisiae $(15,16)$, the Golgi and plasma membrane pools of PI(4)P are functionally distinct, i.e. Golgi PI(4)P does not substantially contribute to plasma 
membrane PI(4)P. A previous study suggested that the plasma membrane type III $\alpha$ PI4kinase Stt4 was not essential for viability in C. albicans (17), raising the possibility that plasma membrane PI(4)P may be dispensable.

In yeast and mammalian cells, Stt4 is part of a complex comprised of the membrane protein Efr3 and a scaffold protein Ypp1 (TTC7 in mammals). Efr3 and Ypp1 are required for both targeting of Stt4 to the plasma membrane and PI-4-kinase activity (18-20). Specifically, Efr3 is critical for the plasma membrane association of the PI-4kinase complex and Ypp1/TTC7 has been shown to bind directly both Efr3 and Stt4 (18, 19), suggesting a scaffolding function. In S. cerevisiae, both Ypp1 and Efr3 are essential for viability $(18,21,22)$, whereas in $S$. pombe only Ypp1 is essential for viability (8). In conditional yppl and efr3 S. cerevisiae mutants, cellular PI(4)P levels were reduced approximately two-fold $(18,20,22)$.

To investigate the importance of plasma membrane PI(4)P in C. albicans hyphal growth and virulence we have generated deletion mutants of efr 3 and $y p p l$, in addition to stt4. These mutants were all viable and had different levels of plasma membrane PI(4)P. Surprisingly, C. albicans cells with little to no plasma membrane PI(4)P remain viable and can proliferate by budding growth. Furthermore, our results indicate that plasma membrane PI(4)P is critical for hematogenously disseminated candidiasis (HDC) with a yppl mutant exhibiting reduced virulence and an stt4 mutant causing no lethality, consistent with their plasma membrane PI(4)P levels. Our analyses of the cell wall suggest that the dramatic reduction in virulence in HDC is, in part, due to an unmasking of cell surface $\beta(1,3)$-glucan. 


\section{Results}

The non-essential Stt4 PI-4-kinase complex is critical for invasive filamentous growth and cell wall integrity

Previously, we have generated strains in which the expression of STT4 could be repressed with the addition of doxycycline (14). In the presence of this repressor, there was approximately a 10-fold decrease in STT4 transcript levels and budding growth was similar to that of the wild-type. When STT4 was repressed, this mutant was able to undergo invasive filamentous growth in response to serum, yet invasive filaments emanating from mutant colonies were $\sim 4$-fold shorter compared to wild-type and complemented strains. Upon repression of STT4 in liquid media containing serum, the cells elongated with protrusions that were roughly $1 / 3$ the length of wild-type cells after 2 hours at $37^{\circ} \mathrm{C}$. However, in these repression conditions, the stt4 mutant still expressed STT4 and plasma membrane PI(4)P was still detected (14). We also generated a 'decreased abundance by mRNA perturbation' (DAmP) allele (23) in C. albicans (24), by constructing strains in which one copy of STT4 was deleted and a dominant nourseothricin resistance marker (SAT1) was integrated just 3' of the STT4 stop codon. These DAmP mutants had between 2-4-fold reduction in STT4 transcript levels compared to a wild-type strain, yet filamentous growth was indistinguishable from the wild-type in liquid and on solid media containing fetal calf serum (Figure S1). More recently, a stt4 deletion mutant was isolated in a screen for mutants exhibiting hypersensitivity to Hsp90 inhibition via geldanamycin, with aberrant filamentation in the presence of geldanamycin or RPMI (17).

To determine if plasma membrane PI(4)P is essential, we attempted to delete this PI-4-kinase, as well as the two other putative components of the Stt4 complex, Efr3 and Ypp1. To generate a stt4 deletion mutant, we removed the remaining STT4 copy in the 
DAmP mutant, by taking advantage of the 3' SAT1 marker to target homologous recombination. Southern blotting, as well as PCR of genomic DNA (gDNA), confirmed the absence of STT4 in this homozygous deletion strain (Figure S2A, S2B). In addition, we were able to generate homozygous deletion mutants of EFR3 and $Y P P 1$, which were verified by PCR (Figure S3A). RT-PCR revealed the complete absence of STT4 mRNA transcript, as well as that of EFR3 and YPP1 in the respective mutants (Figure S2C, S3B). Interestingly, the levels of mRNA transcripts of other PI- and PIP-kinases and phosphatase, including $P I K 1, M S S 4$ and $S A C 1$ were unaffected in the stt4 deletion mutant (Figure S2C). These strains all grew with doubling times that were indistinguishable from that of the wild-type strain $(81 \pm 6 \mathrm{~min}$ for the wild-type strain compared to $88 \pm 7 \mathrm{~min}$ for $s t t 4 \Delta / s t t 4 \Delta ; 86 \pm 5 \mathrm{~min}$ for $s t t 4 \Delta /$ stt4 $\Delta+S T T 4 ; 86 \pm 2$ min for efr $3 \Delta /$ efr $3 \Delta ; 89 \pm 1$ min for $y p p 1 \Delta / y p p 1 \Delta)$, indicating that the PI-4-kinase complex is not necessary for viability or yeast-phase growth in C. albicans.

In the presence of serum, however, we observed a striking filamentous growth defect (Figures 1 and 2) in the efr 3, yppl and stt4 deletion mutants. The mutants appeared to form short germ tubes but were unable to form longer hyphal filaments. Similarly, all three strains were completely defective in invasive growth agar media containing fetal calf serum (Figure 1C and 2C). These defects were complemented by the reintroduction of a copy of the respective genes, which was confirmed by PCR of gDNA and RT-PCR of mRNA transcripts (Figure S2B, S2C, S3A, S3B). A stt4 allele that is a hypomorph with reduced in vivo lipid kinase activity (25) was identified in S. cerevisiae in a genetic screen for aminophospholipid transport mutants (7). Hence, we also examined whether such a mutant, in which a highly conserved amino acid in the catalytic domain, Gly 1782 was changed to Asp (in C. albicans G1810D), was critical for hyphal growth. Figure 1B shows that a strain expressing as a sole copy this mutated version of STT4 exhibited 
defects in filamentous growth that were intermediate between the stt4 deletion mutant and the complemented strain. Furthermore, compared to the wild-type and complemented strains, the efr 3, yppl and stt4 deletion mutants did not grow in the presence of the cell wall perturbants, including the antifungal drug caspofungin, calcofluor white and Congo Red (Figure 3). A lower concentrations of caspofungin (50 ng/mL compared to 125 $\mathrm{ng} / \mathrm{mL}$ ) revealed that the stt4 deletion mutant exhibits the greatest sensitivity to this antifungal drug, with the ypp 1 deletion mutant exhibiting intermediate sensitivity and efr 3 deletion mutant having the lowest sensitivity (Figure S4A). These mutants were however not temperature sensitive and grew similar to wild-type controls at $30^{\circ} \mathrm{C}$ and $37^{\circ} \mathrm{C}$ (Figure S4B) and, in contrast to what has been previously reported (26), they did not exhibit an increased sensitivity to fluconazole. Given the effects of cell wall perturbants, we examined the cell wall thickness and composition in the stt4 homozygous deletion mutant. Figures 4A and 4B show that, in this PI-4-kinase deletion mutant, the cell wall was on average $50 \%$ thicker compared to wild-type cells. Flow cytometry was used to quantitate the exposed $\beta(1,3)$-glucan, as well as the total chitin, mannan and glucan. A significant increase in exposed $\beta(1,3)$-glucan, mannan and glucan content was observed in the stt4 deletion mutant compared to the wild-type strain (Figure 4C). On average stt4 mutant cells had a $30 \%$ increase in exposed $\beta(1,3)$-glucan and a $60 \%$ in mannan and glucan content. This cell wall integrity defect and increase in cell surface exposure of $\beta(1,3)$-glucan are reminiscent of that observed in the phosphatidylserine synthase mutant chol $\Delta /$ chol $\Delta$ (27-30). Together, our results show that the PI-4-kinase complex is critical for filamentous growth and cell wall integrity in C. albicans, specifically masking cell wall $\beta(1,3)$-glucan. 
The defect in filamentous growth in mutants for all three components of the PI-4-kinase complex suggested that plasma membrane $\mathrm{PI}(4) \mathrm{P}$ is critical for this process. Therefore, we examined the in vivo $\mathrm{PI}(4) \mathrm{P}$ levels using a fluorescent reporter that binds preferentially this acidic phospholipid at the plasma membrane (13). This reporter can also bind PI(4)P at the Golgi and we have previously shown that, upon a reduction in plasma membrane $\mathrm{PI}(4) \mathrm{P}$, the reporter relocalizes to the Golgi (13). In wild-type cells, we observed this GFP-PH ${ }^{\mathrm{OSH}}-\mathrm{PH}^{\mathrm{OSH}}$-GFP reporter localize predominantly at the plasma membrane, yet in efr 3, yppl and stt4 mutants there was a decrease in plasma membrane PI(4)P, with a concomitant increase in intracellular Golgi PI(4)P signal (Figure 5A). Quantification of the reporter fluorescence from these central z-sections, using the Matlab program HyphalPolarity (14), indicate that the ratio of mean plasma membrane signal to mean internal signal decreased progressively in the efr 3, ypp 1 and stt4 mutants, resulting from the decrease in plasma membrane PI(4)P together with the overall increase in internal PI(4)P levels (Figure 5B). Closer examination of stt4 mutant cells revealed little to no plasma membrane PI(4)P (Figure 5A) and this was confirmed by quantification of the normalized fraction of total signal at the plasma membrane, i.e. the plasma membrane signal excluding the Golgi cisternae divided by the total cell signal excluding the Golgi cisternae (Figure 5C). The average normalized plasma membrane PI(4)P in stt4 mutants was very close to $0(0.078 \pm 0.016)$, with $60 \%$ of cells $(n=200)$ having undetectable plasma membrane PI(4)P. Together, these results indicate that $C$. albicans cells with undetectable plasma membrane $\mathrm{PI}(4) \mathrm{P}$ are viable and suggest that this plasma membrane acidic phospholipid is specifically critical for filamentous growth.

Previously we have shown that a reduction in Golgi PI(4)P results in Golgi proliferation (13). Hence, we examined whether the reduction of plasma membrane PI(4)P observed in efr 3, yppl and stt4 deletion mutants affected the Golgi. While there 
was a small decrease (20-25\%) in the number of Golgi cisternae per cell in efr 3 and yppl mutants compared to the wild-type (Figure 6A), there was no difference in the number of Golgi cisternae per cell in the stt4 mutant (Figure 6B). These results further confirm that Golgi and plasma membrane PI(4)P pools are functionally distinct. Our previous analyses of a STT4 repressible strain revealed that upon a 10-fold repression of STT4 transcript, there was a reduction of plasma membrane $\mathrm{PI}(4,5) \mathrm{P}_{2}(14)$. As a result, we examined plasma membrane $\mathrm{PI}(4,5) \mathrm{P}_{2}$ levels in the efr 3 , yppl and stt4 deletion mutants. Figure 7 shows that this phosphatidylinositol phosphate was observed at the plasma membrane in all three mutants. Compared to the wild-type strain, there was a reduction of $10-15 \%$ in the plasma membrane $\mathrm{PI}(4,5) \mathrm{P}_{2}$ levels to internal ratios in the efr3, yppl and stt4 mutants. Comparison of the fraction of plasma membrane $\mathrm{PI}(4,5) \mathrm{P}_{2}$ signal revealed a small $(10 \%$ or less) reduction in the stt4 mutants with respect to the wild-type strain, similar to our previous observations with a repressible strain (14). Together these results demonstrate that the dramatic reduction of plasma membrane PI(4)P does not alter Golgi PI(4)P nor does it substantially alter plasma membrane $\mathrm{PI}(4,5) \mathrm{P}_{2}$.

Oxysterol binding proteins, such as Osh6 and Osh7 in S. cerevisiae, are lipid transfer proteins that transfer phosphatidylserine (PS) from the ER to the plasma membrane concomitant with transfer of PI(4)P from the plasma membrane to the ER, where it is hydrolyzed by Sac1 (31-33). In C. albicans, as in S. cerevisiae, Sac1, which localizes to the ER and Golgi, is critical for regulating plasma membrane PI(4)P levels $(13,34,35)$. Given that in the efr 3, yppl and stt4 deletion mutants there was a reduction in PI(4)P levels, we examined whether PS plasma membrane levels were affected using a fluorescent reporter that binds preferentially this acidic phospholipid (36). In wild-type cells we observed this LactC2-GFP reporter localized predominantly at the plasma membrane with little to no internal signal observed (Figure 8A). In contrast, in the efr3, 
yppl and stt4 mutants, peri-nuclear signal was observed, characteristic of the ER, yet plasma membrane PS was still apparent in each of these mutants (Figure 8A). Quantification of signals from central z-sections using the Matlab program HyphalPolarity (14) confirmed that the ratio of mean plasma membrane signal to mean internal signal decreased progressively in the efr $3, y p p 1$ and stt 4 mutants, resulting in part, from a progressive increase in internal PS signal (Figure 8B). Similarly, the mean plasma membrane PS fraction also decreased progressively in the efr $3, y p p 1$ and stt 4 mutants, with the stt4 deletion mutant exhibiting an approximately $50 \%$ reduction compared to wild-type cells. The stt4 hypomorph, Stt4[G1810D], had plasma membrane PS levels intermediate between the efr 3 and yppl mutants. Together, these results suggest that sufficient PI(4)P is critical for the transport of PS from the ER to the plasma membrane. We next examined if there was a correlation between the plasma membrane PI(4)P and PS levels. Figure 9 shows that there is a direct correlation between the levels of these two lipids in the different deletion mutants and the wild-type strain. Plasma membrane lipid levels in the Stt4[G1810D] mutant were also consistent with such a correlation. Note that while we were unable to detect plasma membrane PI(4)P in the stt4 deletion strain, approximately $50 \%$ of the plasma membrane PS was detectable in this mutant. Furthermore, plasma membrane PS levels in the efr 3 mutant were not dramatically different from that of the wild-type cells. Together, our results suggest that the filamentation and cell wall integrity defects observed in the three Stt4 complex mutants are likely to be due to lack of plasma membrane PI(4)P and not PS.

\section{Stt4 PI-4-kinase complex localizes to cortical patches}

To determine the distribution of Efr3, Ypp1 and Stt4, we generated 3X-mScarlet fusions by tagging the chromosomal copy of the respective genes. These fusion were functional 
in that as a sole copy they complemented the cell wall integrity defect of the respective mutants (Figure S5). Despite the low abundance of these Stt4 PI-4-kinase complex subunits, we observed patches around the cortex of the mother cell and buds (Figure 10A), which were also visible along the cortex of the germ tubes with reduced signals in the mother cell (Figure 10B). In S. cerevisiae, Ypp1 and Efr3 are critical for Stt4 membrane localization. Here, we observed that while Stt4 localization to the cortex is dependent on Ypp1 in C. albicans, in the efr 3 mutant there were still some cells with cortex localized Stt4 (Figure 10C). Efr3 cortex localization depended upon Ypp1 and Stt4, with loss of cortex signal observed in either mutant. In contrast, there were some cells with Ypp1 cortex signal in the efr 3 mutant, but not in the stt 4 mutant. In this efr 3 mutant $40-50 \%$ of cells exhibited punctate localization of Stt4 and Ypp1. In the ypp1 mutant, we did not observe cells with either Stt4 or Efr3 localized. Finally, in the stt4 mutant, only 10\% of cells exhibited punctate localization of Ypp1. RT-PCR revealed that the mScarlet transcript levels in all mutants were similar to that of the wild-type (Figure S6). As these results suggested that Ypp1 and Stt4 were the most critical components of this PI-4-kinase complex, we examined whether these proteins colocalized to the same cortical patches. Figure S7 shows that, in a strain expressing Ypp1-mTurquoise and Stt4-3xmScarlet, there was only limited colocalization of these two proteins, suggesting that, if they form a complex, it is likely to be transient.

\section{Plasma membrane PI(4)P is critical for virulence}

To investigate the importance of plasma membrane PI(4)P in virulence, we examined the efr3, yppl and stt4 mutants in two murine infection models, HDC and oropharyngeal candidiasis (OPC). As C. albicans responds to cues, such as the presence of serum, by the induction of hyphal specific genes (HSG), many of which are critical for virulence, 
we initially examined HSG levels after 30 and 120 min incubation with serum. Figure 11A shows that the stt4 deletion mutant induced a range of HSGs, including ECE1, HGC1, HWP 1, ALS3 and SAP4-6, $10^{3}-10^{5}$-fold (excluding SAP4-6), similar to the wildtype strain, with only a 5-fold reduction in induced transcript levels observed after $30 \mathrm{~min}$ incubation. In the HDC model, $20 \%$ and $100 \%$ of mice infected with the $y p p l$ and stt4 mutants, respectively, survived 2 weeks after injection, while all of the mice infected with the efr 3 mutant or wild-type strain died within 5-6 days (Figure 11B). The virulence was significantly restored in complemented strains (Figure 11B). Furthermore, we examined whether the stt4 deletion mutant could filament after long incubation times in serum or in kidney homogenate (37). After $6 \mathrm{Hr}$ incubation with either serum or kidney homogenate, despite observing elongated cells, the stt4 mutant did not form hyphal filaments, compared to wild-type and complemented strains (Figure S8). It is unlikely that the unmasking of cell surface $\beta(1,3)$-glucan and these virulence defects are due to altered plasma membrane PS levels as a cho1 $1 /$ cho1 1 ::CHO1 strain with greater than $50 \%$ reduction in PS levels had no increase in unmasked $\beta(1,3)$-glucan and exhibited full virulence in mouse models of systemic and oropharyngeal infection (27-29). In the OPC model, only the ypp 1 mutant was substantially less virulent than the wild-type strain, with the oral fungal burden of the infected mice reduced by $\sim 25$-fold (Figure 11C). Nonetheless, examination of the histopathology of tongue thin sections revealed that in infection lesions, all strains were able to filament (Figure S9). Together, these data suggest that plasma membrane PI(4)P is required for virulence during hematogenously disseminated candidiasis, with a decrease in virulence observed upon a $60 \%$ reduction in plasma membrane PI(4)P and the lack of lethality observed in the absence of this phosphatidylinositol phosphate. Our data also indicate that only a small amount of plasma membrane PI(4)P is required for normal virulence during oropharyngeal candidiasis. 


\section{Discussion}

Our results show that plasma membrane $\mathrm{PI}(4) \mathrm{P}$ is critical for the C. albicans yeast to filamentous growth transition and cell wall integrity. We show that all three members of the Stt4 PI-4-kinase complex are dispensable for viability, yet are required for filamentous growth and cell wall integrity. Furthermore, quantitative analyses indicate that these mutants have decreasing levels of plasma membrane PI(4)P, going from efr3 to yppl to stt4; a majority of the stt4 cells lack detectable PI(4)P at the plasma membrane. In addition, a stt4 hypomorph (25) had similar plasma membrane PI(4)P levels as the efr3 mutant. We observed little to no alteration in Golgi PI(4)P and plasma membrane $\mathrm{PI}(4,5) \mathrm{P}_{2}$ in these Stt4 PI-4-kinase complex mutants. Consistent with a link between plasma membrane PI(4)P and PS, there is a gradual increase in internal pools of PS in efr3, yppl and stt4 mutants, yet even in the stt4 mutant that lacks plasma membrane PI(4)P, PS is still detected at the plasma membrane. All three of these PI-4-kinase complex proteins localize to cortical patches but only Ypp1 and Stt4 appear to be critical for the complex formation. Furthermore, our results reveal that plasma membrane PI(4)P is important for masking cell surface $\beta(1,3)$-glucan, but not for induction of a number of hyphal specific genes. Plasma membrane $\mathrm{PI}(4) \mathrm{P}$ is critical for pathogenicity during hematogenously disseminated candidiasis, but less so for oropharyngeal candidiasis, suggesting that this lipid has different roles in distinct anatomic infection sites, which we attribute, in part, to host immune recognition via unmasked cell surface $\beta(1,3)$-glucan.

Using strains in which either the C. albicans PI-4-kinase, i.e. Pik1 at the Golgi or Stt4 at the plasma membrane, could be repressed, we previously showed that the Golgi PI-4-kinase is strictly required for invasive filamentous growth (13), whereas repression of the plasma membrane PI-4-kinase mutant resulted in cells that can still form short protrusions and invasive filaments (14). This repressible stt4 mutant had an $\sim 10$-fold 
reduction in STT4 transcript levels compared to a wild-strain, yet PI(4)P was still detectable at the plasma membrane. To address the importance of plasma membrane PI(4)P in C. albicans, we generated homozygous deletion mutants in the PI-4-kinase complex, comprised of Efr3, Ypp1 and Stt4. None of these PI-4-kinase components are essential for viability in C. albicans in contrast to other fungi, specifically in S. cerevisiae where both Pik1 and Stt4 are essential $(6,7,38,39)$. We speculate that the plasma membrane $\mathrm{PI}(4) \mathrm{P}$ is required to maintain filamentous growth in $C$. albicans via contributions to cell polarity and membrane traffic. A. nidulans and C. neoformans appear to only have a Stt4 PI-4-kinase, that is essential for viability $(4,5)$. In addition, although C. albicans stt4, ypp1, and efr 3 mutants showed increased sensitivity to cell wall perturbants, they did not exhibit a temperature sensitive growth defect, as was the case in S. cerevisiae where a temperature sensitive stt4 mutant lysed at the non-permissive temperature (15). Our quantitative analyses revealed that the majority of C. albicans cells lacking Stt4 have undetectable levels of PI(4)P at the plasma membrane, suggesting that this lipid is dispensable for viability. Such C. albicans mutants with little to no plasma membrane PI(4)P are sensitive to cell wall perturbants and have thicker cell walls with increased levels of glucan and mannan, along with an increase in cell surface exposed $\beta(1,3)$-glucan, indicating that $\mathrm{PI}(4) \mathrm{P}$ is critical for maintaining cell wall integrity. We speculate that plasma membrane glucan synthase activity is regulated by $\mathrm{PI}(4) \mathrm{P}$, via the Rho1 regulatory subunit (40). This raises the question of how $\mathrm{PI}(4,5) \mathrm{P}_{2}$ is generated in the stt4 deletion mutant and we speculate that PI(4)P from the Golgi may be phosphorylated by Mss4. This could occur via Mss4 localization to the Golgi or secretory vesicles, although this kinase has not been detected in these compartments in C. albicans (14). Alternatively, a small amount of Golgi PI(4)P could reach the plasma membrane and be immediately phosphorylated. 
Previous studies in S. cerevisiae indicated that the levels of PS and plasma membrane PI(4)P are linked. For example, an S. cerevisiae stt4 mutant with reduced levels of plasma membrane PI(4)P accumulates PS (7, 25). Furthermore a S. cerevisiae sacl phosphatase mutant that results in a dramatic increase in $\operatorname{PI}(4) \mathrm{P}(34,35)$ has decreased PS levels at the plasma membrane, with an increase in intracellular membranes (41). Similarly, in fibroblasts, PI-4-kinase III $\alpha$ knockouts have decreased plasma membrane PS (42). The S. cerevisiae oxysterol proteins Osh6 and Osh7 have been shown to exchange PS for PI(4)P in vitro, and in vivo a sacl mutation resulted in a redistribution of added lyso-PS, which is normally at the plasma membrane, to the ER (32). It was proposed that a PI(4)P gradient from the plasma membrane to the ER drives PS transport via Osh6/7 from the ER to the plasma membrane (32). Given that C. albicans cells are viable with little to no plasma membrane $\mathrm{PI}(4) \mathrm{P}$, it is likely that this so-called 'phosphoinositide-motive force' is not essential (43). Nonetheless, we observed a progressive increase in intracellular PS in C. albicans mutants with decreasing plasma membrane PI(4)P, demonstrating the plasma membrane PI(4)P is critical for PS transport to the plasma membrane. This result indicates that Osh6/7 counter-transporters account for roughly half of the plasma membrane PS and suggests the remaining PS may be delivered to the plasma membrane via vesicular traffic.

In S. cerevisiae, Stt4 and Ypp1 and Efr3 and Ypp1 colocalize in cortical patches (18) and the latter interaction has also been observed by bimolecular fluorescence complementation (44). In mammalian cells and fission yeast, Stt4, Ypp1 and Efr3 also form a complex observed by co-immunoprecipitation and co-localization $(8,19,45,46)$. Here, we show that all three Stt4 PI-4-kinase complex proteins localize to cortical patches during budding and hyphal growth, yet we did not observe substantial co-localization between Ypp1 and Stt4 proteins, although each protein was critical for the cortical patch 
localization of the other protein. This suggests that Yppl is important for targeting and/or stabilization of the Stt4 PI-4-kinase, as has been observed in S. cerevisiae (18). Interestingly, although Efr3 is important for cortical patch localization of Ypp1 and Stt4 proteins, it is not absolutely required as in S. cerevisiae (18). These results suggest that Efr3 facilitates targeting and/or stabilization of the complex, but that plasma membrane PI-4-kinase activity is not strictly dependent on it.

Cell wall defects in mutants lacking the PS synthase Cho1, that have little to no PS, are in some respects similar to those of stt4 deletion mutant cells. For example, a $C$. albicans chol deletion mutant also had a thicker cell wall and exhibited increased sensitivity to the antifungal drug caspofungin (27). However, this chol mutant had a dramatic increase in cell wall chitin levels $(27,30,47)$, in contrast to a mutant lacking plasma membrane PI(4)P. Interestingly, both stt4 and chol deletion mutants exhibited an increase in exposed cell wall $\beta(1,3)$-glucan, with the latter mutant having a roughly 10 fold greater increase in this polysaccharide (30) compared to the stt4 mutant. However, given that a chol $\triangle /$ chol $\triangle:$ CHO1 strain with $>50 \%$ reduction in PS levels had no increase in unmasked $\beta(1,3)$-glucan $(27,28)$, it is unlikely that the cell wall defects observed in the mutant lacking plasma membrane PI(4)P is due to the reduced PS levels.

The importance of lipids with respect to $C$. albicans virulence has been challenging to determine, as a number of lipids are essential or viability and cell growth. One lipid that has been shown to be critical for pathogenicity in a range of different fungi is the sphingolipid glucosylceramide (48-51). Furthermore, mutants lacking either the PS synthase Cho1 or PS decarboxylases (Psd1 and Psd2) were avirulent in murine models of systemic candidiasis and oropharyngeal candidiasis $(27,29)$. However, both of these mutants grew substantially slower than wild-strains and exhibited a $\sim 50 \%$ reduction in phosphatidylethanolamine (PE) levels (27). Expression of a heterologous serine 
decarboxylase revealed that the observed virulence defects, as well as $\beta(1,3)$-glucan unmasking, were in fact due to the ethanolamine auxotrophy of these mutants (29). Using an in vitro assay for host pathogen interactions, an $\sim 2$-fold reduction in macrophage lysis was observed with a stt4 mutant (17), suggesting that this kinase may be important for virulence. Similarly, the filamentation defect and increased exposure of cell surface $\beta(1,3)$-glucan in the plasma membrane PI-4-kinase deletion mutant indicated that PI(4)P has a role in virulence. Interestingly, a recent study by Dunker et al. showed that rapid proliferation can compensate for the absence of filamentation in a murine model of systemic candidiasis, directly challenging the idea that filamentation is strictly required for virulence (37). Of the three Stt4 PI-4-kinase complex mutants, stt4, and to a lesser extent ypp1, exhibited virulence defects in a murine model of systemic candidiasis. Given that all Stt4 PI-4-kinase complex mutants are defective in filamentation, this decrease in virulence is unlikely to be attributable to a defect in morphogenesis. Indeed, elongated stt4 mutant cells were observed after long incubation times with serum or kidney homogenate, and hyphal gene induction was not substantially different from the wildtype strain after two hours incubation in serum. Furthermore, in a murine model for oropharyngeal candidiasis, all Stt4 PI-4-kinase complex mutants were able to filament. Our results, however, indicate a correlation between PI(4)P at the plasma membrane and systemic candidiasis, given the yppl mutant and the stt4 mutant have a 3-fold or greater decrease in plasma membrane PI(4)P. As the Stt4 PI-4-kinase complex mutants all have altered cell wall integrity, substantial alterations in the level of plasma membrane PI(4)P lead to corresponding changes in the cell wall, in particular, exposure of masked $\beta(1,3)$ glucan that is recognized by host immune cells, ultimately contributing to the reduction in virulence in the systemic candidiasis model. Consistent with this explanation, the stt4 deletion mutant exhibited little to no defect in OPC infection assay, in which the host 
immune response is less critical. It will be interesting to further analyze the cell wall defects in these Stt4 PI-4-kinase complex mutants given their different levels of plasma membrane PI(4)P, which will be useful tools in dissecting how plasma PI(4)P regulates the cell wall during fungal infection. 


\section{Acknowledgements}

We thank J. Wendland, S. Bates and A. Mitchell for strains and plasmids. We thank S. Bogliolo for assistance. We thank the Platforms Resources in Imaging and Scientific Microscopy facility (PRISM; M. Mondin, S. Lachambre, B. Monterroso), the Cytometry Platform (BV-CyAn; A. Loubat), the Experimental Histopathology Platform (S. Rekima) and Microscopy Imaging Côte d'Azur (MICA) for microscopy, cytometry and histopathology support. This work was supported by the CNRS, INSERM, Université Côte d'Azur and ANR (ANR-11-LABX-0028-01, ANR-16-CE13-0010-01 and ANR-19CE13-0004-01) grants, by grant R01DE026600 from the NIH USA and grant SAF201786192 from Spanish Ministry from Science and Innovation. 


\section{Materials and Methods}

\section{Strain and plasmid construction}

Standard methods were used for $C$. albicans cell culture, molecular and genetic manipulations. Strains were grown in yeast extract-peptone dextrose (YEPD) at $30^{\circ} \mathrm{C}$ unless otherwise indicated and induction of filamentous growth was carried out with $50 \%$ serum at $37^{\circ} \mathrm{C}$ for $90 \mathrm{and} /$ or $120 \mathrm{~min}$. To determine doubling times cells were grown in YEPD media at $30^{\circ} \mathrm{C}$ and optical density was followed over $8 \mathrm{Hr}$ of logarithmic growth. For induction with kidney homogenates, kidneys from male C57BL/6 mice were aseptically removed, dounce homogenized with sterile PBS $(0.4 \mathrm{~g} / \mathrm{mL})$ and this homogenate was diluted 1:1 with logarithmically growing C. albicans strains in YEPD. Serial dilutions of the different strains on YEPD plates containing Congo red (400 $\mu \mathrm{g} / \mathrm{mL})$, Calcofluor White $(25 \mu \mathrm{g} / \mathrm{mL})$, Caspofungin (50 and $125 \mathrm{ng} / \mathrm{mL}$ ), and Fluconazole $(10 \mu \mathrm{g} / \mathrm{mL})$ were examined after 2-3 days of incubation at $30^{\circ} \mathrm{C}(52)$. The strains and plasmids used are listed in Tables S1 and S2, respectively and the oligonucleotides used are listed in Table S3. All strains are based on BWP17 background (53). The efr $3 \Delta /$ efr $3 \Delta$ and ypp $1 \Delta$ ypp $1 \Delta$ strains were generated by homologous recombination. Each copy was replaced by either HIS1 or URA3 using knockout cassettes generated by amplification from pGemHIS1 and pGemURA3 (53) with primers $\mathrm{CaEfr} 3 \mathrm{pKO} / \mathrm{CaEfr} 3 \mathrm{mKO}$ and CaYpp1pKO/CaYpp1mKO. In order to generate prototrophic strains, the pExpARG plasmid was linearized with StuI and integrated in RPS1 locus. pExpARG-pEFR3-EFR3 and pExpARG-pYPP1-YPP1 plasmids were constructed by amplification of gDNA using primers with a unique XhoI site at 5 ' and a unique NotI site at 3' ends, with $1 \mathrm{~kb}$ upstream and downstream of the respective ORFs. These fragments were subsequently cloned in pExpARG yielding pExpARG-pEFR3EFR3 and pExpARG-pYPP1-YPP1, respectively. Finally, pExpARG-pEFR3-EFR3 and 
pExpARG-YPP1-YPP1 were integrated in the RPS1 locus yielding the recovery strains

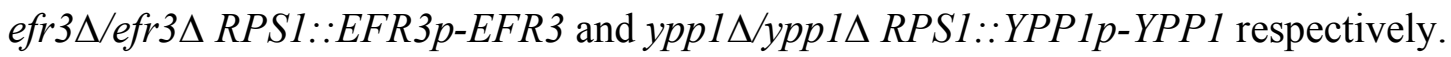

To generate mutants, we first generated a stt4 DAmP (Decrease Abundance by mRNA Perturbance) allele by integration of the SAT1 gene (via amplification of pFASAT1 (54) with primers CaSATDAmPpS1 and CaSATDAmPmS2) 5' of the STT4 stop codon. The remaining STT4 copy was replaced with URA3 using a knockout cassette generated from amplification of pGemURA3 (53) with primers CaStt4pKO and CaStt4mKO. As the stt4 $\Delta /$ stt4DAmP strain behaved identical to the wild-type, we next generated a stt4 $\Delta /$ stt4 $\Delta$ strain (PY4377) by replacing the allele stt4DAmP with HIS1 using a knockout cassette amplified from pGemHIS1 (53) with primers CaStt4pKO and CaSATDAmPmKO. The URA3 gene of the stt4L/stt4A strain (PY4377) was then replaced by $S A T 1$ which was amplified from pGFP-Nat (55) using primers CaURApSAT and CaURAmSAT, so that URA3 could be subsequently integrated at the RPS1 locus for murine HDC assays. To generate this $\mathrm{URA}^{+}$strain, pExpURA3 (56) was linearized and integrated in RPS1 locus yielding PY5040, which was subsequently rendered $\mathrm{ARG}^{+}$by transformation with linearized pExpARG plasmid, yielding PY5111. In order to reintegrate STT4 at the STT4 locus, we generated a pSTT4-STT4-STT4t cassette in which unique AscI and PmeI sites were inserted into the STT4 terminator (623 bp 3' of the stop codon) by site directed mutagenesis using primers CaStt4term_mAscIPmeI and CaStt4term_pAscIPmeI. Subsequently ARG4 was amplified from pFaARG4 plasmid using primers CaARG4AscI-S1 and CaARG4PmeI-S2, yielding plasmid pExpARGSTT4-STT4-STT4t::ARG4. To generate the recovered strain, the pSTT4-STT4STT4t::ARG4 fragment (digested by XhoI and NotI) from this plasmid was used to replace stt4::HIS1 in PY5040, resulting in $s t t 4 \Delta$ stt4A::STT4, PY5119. To generate a prototroph recovered strain HIS1 was added back using linearized pGemHIS1 resulting 
in PY5131. To generate the hypomorph stt4 mutant which encodes Stt4[G1810D], site directed mutagenesis was carried out with pExpARG-pSTT4-STT4-STT4t::ARG4 using primers CaStt4G1810DpEcoRV and CaStt4G1810DmEcoRV, resulting in pExpARGSTT4p-STT4*-STT4t::ARG4, which was subsequently digested with XhoI and NotI and transformed into PY5040 resulting in PY5757.

Plasmids containing the phospholipid reporters for plasma membrane PI(4)P, Golgi PI(4)P, PI(4,5)P 2 and PS, pExpARG-pADH1-GFP-(PH $\left.{ }^{\mathrm{OSH} 2[\mathrm{H} 340 \mathrm{R}]}\right)_{2}-\mathrm{GFP}$, pExpARG-pADH1-PH $\left.{ }^{\mathrm{FAPP} 1[\mathrm{E} 50 \mathrm{~A},} \quad \mathrm{H}^{\mathrm{H} 54 \mathrm{~A}}\right]_{-} \mathrm{GFP} \quad(13), \quad$ pExpARG-pADH1-GFP-PH ${ }^{\mathrm{Plc} \delta}$ $\mathrm{PH}^{\mathrm{Plc} \delta}$-GFP (14) and pExpARG-pACT1-GFP-yeLactC2 (36), respectively, were linearized and integrated into the RPSI locus. For expressing these reporters in the Stt4[G1810D] strain (PY5757), the ARG4 gene was replaced by SAT1 using the primers CaArgExchS1 and CaArgExchS2 and pFaSAT1 (54). To generate fluorescent protein fusions with Stt4, Efr3, and Ypp1 either 3x-mScarlet or mTurquoise2 was amplified using primers CaStt4pXFPS1 and CaStt4mXFPS2, CaEr3pXFPS1 and CaEr3mXFPS2, CaYpp1pXFPS1 and CaYpp1mXFPS2 respectively and plasmids pFA-3x-mSc-ARG4 (57), pFA-3x-mSc-CdHIS1 or pFA-mTurq2-ARG4 (C. Puerner, M. Bassilana, and R. A. Arkowitz, in preparation).

\section{Southern blot analyses; RT-PCR; qRT-PCR; and chitin staining}

For Southern analysis, EcoRV digested gDNA was separated on a 1\% agarose gel, transferred to a nylon membrane and fixed by UV crosslinking as described (36). The hybridization probes were generated by PCR using primers (STT4: CaStt4p5199 and CaStt4m5543; URA3: CaUra3pXhoI and CaURA3m81) and Amersham ECL Direct Nucleic Acid Labelling and Detection System kit (GE Healthcare UK Limited, Little Chalfont Buckinghamshire, UK) following manufacturer's instructions. For RT-PCR and 
qRT-PCR, primers used (Genep-TM/Genem-TM) are listed in Table S3 and RNA extraction was carried out using Master Pure yeast RNA extraction purification kit (Epicentre) from budding cells and cells incubated with serum. For RT-PCR Actin amplification 30 cycles were used and 32 cycles were used to amplify EFR3, YPP1, STT4, PIKlalpha, MSS4, SAC1 and mSCARLET. qRT-PCR analyses were carried out as previously described (36) with indicated primers (Genep-TM/Genem-TM; Table S3). For chitin staining exponentially growing cells were fixed and stained with $25 \mu \mathrm{g} / \mathrm{ml}$ Calcofluor White solution and imaged using Spinning disk confocal microscope (58).

\section{Quantitation of cell wall components using flow cytometry.}

Logarithmically growing strains were stained for exposed $\beta(1,3)$-glucan using an anti$\beta(1,3)$-glucan mAb (400-2; Biosupplies, Australia) primary antibody and a donkey antimouse $\operatorname{IgG}(\mathrm{H}+\mathrm{L})$ secondary antibody conjugated to Alexa Fluor 568 (A10037; ThermoFisher, France), essentially as described (28). Antibody dilutions, 1:600, primary and 1:500, secondary were used. For total chitin, mannan and glucan, Calcofluor White (Fluorescent Brightener 28 M2R; Sigma), Concanavalin A-Tetramethylrhodamine (11540176; ThermoFisher, France) and Aniline Blue soluble sodium salt (10656822; ThermoFisher, France), were used at concentrations of $25 \mu \mathrm{g} / \mathrm{mL}, 50 \mu \mathrm{g} / \mathrm{mL}$ and 50 $\mu \mathrm{g} / \mathrm{mL}$, respectively. Cells were fixed with 4\% PFA in PBS for all analyses and washed prior to staining. Incubation of cells with ConA was for $30 \mathrm{~min}$ and cells were subsequently washed. Flow cytometry was carried out on a Cell Analyser BD LSRFortessa Sorp using $355 \mathrm{~nm}$ and $561 \mathrm{~nm}$ laser lines with Hoechst Blue (515/30 nm) and PE-Texas Red (600 nm LP; 610/20 nm) filters, respectively. Data were obtained from 100,000 gated events per strain, from 3 independent experiments. 


\section{Microscopic analyses}

For colony morphology analysis plates were incubated for 3-6 days prior imaging (36). mScarlet and mTurquoise fusions were imaged as described $(57,58)$ with 17 x $0.5 \mu \mathrm{m} z-$ sections. Quantitation of plasma membrane and internal mean signals was performed on central z-sections using the Matlab program Hyphal-Polarity (13). Ratios of plasma membrane to internal signals were normalized by the mean wild-type ratio. Plasma membrane fractions of $\mathrm{PI}(4) \mathrm{P}, \mathrm{PI}(4,5) \mathrm{P}_{2}$ and $\mathrm{PS}$ were calculated as the ratio of plasma membrane signal of total signal which was then normalized to the mean wild-type ratio. To represent these values between 1 and $0,0.5$ was subtracted from the normalized ratio (the Matlab program detects first signal going in from ROI above background, which is the cytoplasm when there is no plasma membrane localization, hence a value of 0.5 is the absence of plasma signal) and then multiplied by 2 so that wild-type plasma membrane fraction is 1 and no plasma membrane signal is 0 . Huygens professional software version 18.04 (Scientific-Volume Imaging) was used for deconvolution of image z-stacks using the appropriate settings for the microscope and excitation source. The signal to noise was set to 10, and the background detection was set to auto, unless otherwise stated. All scale bars are $5 \mu \mathrm{m}$, unless otherwise indicated.

\section{Virulence assays}

HDC was induced in $10 \mathrm{Balb} / \mathrm{C}$ mice (Charles Rivers, Italy) per group by injecting the lateral tail vein with an inoculum of $5 \times 10^{5}$ cells (59). Animal body weight was monitored daily and animals were sacrificed by cervical dislocation when they had lost more than $20 \%$ of their weight. OPC was induced in mice that had been immunosuppressed with cortisone acetate using 7-8 mice per strain as previously described (60). A Vectra Polaris 
Slide Scanner was used to scan histopathology of murine tongue thin sections, stained with periodic acid-Schiff stain.

\section{Ethics statements}

All OPC animal experiments were approved by the Institutional Animal Care and Use Committee (IACUC) of the Lundquist Institute at Harbor-UCLA Medical Center. All HDC animal procedures were approved by the Bioethical Committee and Animal Welfare of the Instituto de Salud Carlos III (CBA2014_PA51) and of the Comunidad de Madrid (PROEX 330/14) and followed the current Spanish legislation (Real Decreto 53/2013) along with Directive 2010/63/EU.

\section{Statistical analysis}

Differences in mean signals, ratios and percentage of filaments were analyzed by $t$-test and survival experiments with mice were analyzed by the Kaplan-Meier method (Logrank test) with Graph Pad Prism 8 (La Jolla, CA).

\section{Transmission electron microscopy}

Budding cell samples were processed for electron microscopy and images acquired as described (61). Cell wall thickness was measured from electron micrographs at 3-4 locations around the mother cell and averaged. 


\section{References}

1. Bochud A, Conzelmann A. 2015. The active site of yeast phosphatidylinositol synthase Pis1 is facing the cytosol. Biochim Biophys Acta 1851:629-40.

2. Boura E, Nencka R. 2015. Phosphatidylinositol 4-kinases: Function, structure, and inhibition. Exp Cell Res 337:136-45.

3. Dornan GL, McPhail JA, Burke JE. 2016. Type III phosphatidylinositol 4 kinases: structure, function, regulation, signalling and involvement in disease. Biochem Soc Trans 44:260-6.

4. De Souza CP, Hashmi SB, Osmani AH, Andrews P, Ringelberg CS, Dunlap JC, Osmani SA. 2013. Functional analysis of the Aspergillus nidulans kinome. PLoS One 8:e58008.

5. Lee KT, So YS, Yang DH, Jung KW, Choi J, Lee DG, Kwon H, Jang J, Wang LL, Cha S, Meyers GL, Jeong E, Jin JH, Lee Y, Hong J, Bang S, Ji JH, Park G, Byun HJ, Park SW, Park YM, Adedoyin G, Kim T, Averette AF, Choi JS, Heitman J, Cheong E, Lee YH, Bahn YS. 2016. Systematic functional analysis of kinases in the fungal pathogen Cryptococcus neoformans. Nat Commun 7:12766.

6. Cutler NS, Heitman J, Cardenas ME. 1997. STT4 is an essential phosphatidylinositol 4-kinase that is a target of wortmannin in Saccharomyces cerevisiae. J Biol Chem $272: 27671-7$

7. Trotter PJ, Wu WI, Pedretti J, Yates R, Voelker DR. 1998. A genetic screen for aminophospholipid transport mutants identifies the phosphatidylinositol 4-kinase, STT4p, as an essential component in phosphatidylserine metabolism. J Biol Chem 273:13189-96. 
8. Snider CE, Willet AH, Chen JS, Arpag G, Zanic M, Gould KL. 2017. Phosphoinositide-mediated ring anchoring resists perpendicular forces to promote medial cytokinesis. J Cell Biol 216:3041-3050.

9. Yoshida S, Ohya Y, Goebl M, Nakano A, Anraku Y. 1994. A novel gene, STT4, encodes a phosphatidylinositol 4-kinase in the $\mathrm{PKC1}$ protein kinase pathway of Saccharomyces cerevisiae. J Biol Chem 269:1166-72.

10. Kadosh D. 2019. Regulatory mechanisms controlling morphology and pathogenesis in Candida albicans. Curr Opin Microbiol 52:27-34.

11. Kornitzer D. 2019. Regulation of Candida albicans Hyphal Morphogenesis by Endogenous Signals. J Fungi (Basel) 5.

12. Min K, Neiman AM, Konopka JB. 2020. Fungal Pathogens: Shape-Shifting Invaders. Trends Microbiol 28:922-933.

13. Ghugtyal V, Garcia-Rodas R, Seminara A, Schaub S, Bassilana M, Arkowitz RA. 2015. Phosphatidylinositol-4-phosphate-dependent membrane traffic is critical for fungal filamentous growth. Proc Natl Acad Sci U S A 112:8644-9.

14. Vernay A, Schaub S, Guillas I, Bassilana M, Arkowitz RA. 2012. A steep phosphoinositide bis-phosphate gradient forms during fungal filamentous growth. J Cell Biol 198:711-30.

15. Audhya A, Foti M, Emr SD. 2000. Distinct roles for the yeast phosphatidylinositol 4-kinases, Stt4p and Pik1p, in secretion, cell growth, and organelle membrane dynamics. Mol Biol Cell 11:2673-89.

16. Santiago-Tirado FH, Legesse-Miller A, Schott D, Bretscher A. 2011. PI4P and Rab inputs collaborate in myosin-V-dependent transport of secretory compartments in yeast. Dev Cell 20:47-59. 
17. O'Meara TR, Veri AO, Polvi EJ, Li X, Valaei SF, Diezmann S, Cowen LE. 2016. Mapping the Hsp90 Genetic Network Reveals Ergosterol Biosynthesis and Phosphatidylinositol-4-Kinase Signaling as Core Circuitry Governing Cellular Stress. PLoS Genet 12:e1006142.

18. Baird D, Stefan C, Audhya A, Weys S, Emr SD. 2008. Assembly of the PtdIns 4kinase Stt4 complex at the plasma membrane requires Ypp1 and Efr3. J Cell Biol 183:1061-74.

19. Nakatsu F, Baskin JM, Chung J, Tanner LB, Shui G, Lee SY, Pirruccello M, Hao M, Ingolia NT, Wenk MR, De Camilli P. 2012. PtdIns4P synthesis by PI4KIIIalpha at the plasma membrane and its impact on plasma membrane identity. J Cell Biol 199:1003-16.

20. Wu X, Chi RJ, Baskin JM, Lucast L, Burd CG, De Camilli P, Reinisch KM. 2014. Structural insights into assembly and regulation of the plasma membrane phosphatidylinositol 4-kinase complex. Dev Cell 28:19-29.

21. Rodriguez-Pena JM, Cid VJ, Sanchez M, Molina M, Arroyo J, Nombela C. 1998. The deletion of six ORFs of unknown function from Saccharomyces cerevisiae chromosome VII reveals two essential genes: YGR195w and YGR198w. Yeast 14:853-60.

22. Zhai C, Li K, Markaki V, Phelan JP, Bowers K, Cooke FT, Panaretou B. 2008. Ypp1/YGR198w plays an essential role in phosphoinositide signalling at the plasma membrane. Biochem J 415:455-66.

23. Yan Z, Costanzo M, Heisler LE, Paw J, Kaper F, Andrews BJ, Boone C, Giaever G, Nislow C. 2008. Yeast Barcoders: a chemogenomic application of a universal donorstrain collection carrying bar-code identifiers. Nat Methods 5:719-25. 
24. Finkel JS, Yudanin N, Nett JE, Andes DR, Mitchell AP. 2011. Application of the systematic "DAmP" approach to create a partially defective C. albicans mutant. Fungal Genet Biol 48:1056-61.

25. Wang Y, Yuan P, Grabon A, Tripathi A, Lee D, Rodriguez M, Lonnfors M, Eisenberg-Bord M, Wang Z, Man Lam S, Schuldiner M, Bankaitis VA. 2020. Noncanonical regulation of phosphatidylserine metabolism by a Sec14-like protein and a lipid kinase. J Cell Biol 219.

26. Caplan T, Polvi EJ, Xie JL, Buckhalter S, Leach MD, Robbins N, Cowen LE. 2018. Functional Genomic Screening Reveals Core Modulators of Echinocandin Stress Responses in Candida albicans. Cell Rep 23:2292-2298.

27. Chen YL, Montedonico AE, Kauffman S, Dunlap JR, Menn FM, Reynolds TB. 2010. Phosphatidylserine synthase and phosphatidylserine decarboxylase are essential for cell wall integrity and virulence in Candida albicans. Mol Microbiol 75:1112-32.

28. Davis SE, Hopke A, Minkin SC, Jr., Montedonico AE, Wheeler RT, Reynolds TB. 2014. Masking of beta(1-3)-glucan in the cell wall of Candida albicans from detection by innate immune cells depends on phosphatidylserine. Infect Immun 82:4405-13.

29. Davis SE, Tams RN, Solis NV, Wagner AS, Chen T, Jackson JW, Hasim S, Montedonico AE, Dinsmore J, Sparer TE, Filler SG, Reynolds TB. 2018. Candida albicans Cannot Acquire Sufficient Ethanolamine from the Host To Support Virulence in the Absence of De Novo Phosphatidylethanolamine Synthesis. Infect Immun 86.

30. Tams RN, Wagner AS, Jackson JW, Gann ER, Sparer TE, Reynolds TB. 2020. Pathways That Synthesize Phosphatidylethanolamine Impact Candida albicans 
Hyphal Length and Cell Wall Composition through Transcriptional and Posttranscriptional Mechanisms. Infect Immun 88.

31. Maeda K, Anand K, Chiapparino A, Kumar A, Poletto M, Kaksonen M, Gavin AC. 2013. Interactome map uncovers phosphatidylserine transport by oxysterol-binding proteins. Nature 501:257-61.

32. Moser von Filseck J, Copic A, Delfosse V, Vanni S, Jackson CL, Bourguet W, Drin G. 2015. Phosphatidylserine transport by ORP/Osh proteins is driven by phosphatidylinositol 4-phosphate. Science 349:432-6.

33. Moser von Filseck J, Vanni S, Mesmin B, Antonny B, Drin G. 2015. A phosphatidylinositol-4-phosphate powered exchange mechanism to create a lipid gradient between membranes. Nat Commun 6:6671.

34. Foti M, Audhya A, Emr SD. 2001. Sac1 lipid phosphatase and Stt4 phosphatidylinositol 4-kinase regulate a pool of phosphatidylinositol 4-phosphate that functions in the control of the actin cytoskeleton and vacuole morphology. Mol Biol Cell 12:2396-411.

35. Roy A, Levine TP. 2004. Multiple pools of phosphatidylinositol 4-phosphate detected using the pleckstrin homology domain of Osh2p. J Biol Chem 279:446839.

36. Labbaoui H, Bogliolo S, Ghugtyal V, Solis NV, Filler SG, Arkowitz RA, Bassilana M. 2017. Role of Arf GTPases in fungal morphogenesis and virulence. PLoS Pathog 13:e1006205.

37. Dunker C, Polke M, Schulze-Richter B, Schubert K, Rudolphi S, Gressler AE, Pawlik T, Prada Salcedo JP, Niemiec MJ, Slesiona-Kunzel S, Swidergall M, Martin R, Dandekar T, Jacobsen ID. 2021. Rapid proliferation due to better metabolic 
adaptation results in full virulence of a filament-deficient Candida albicans strain. Nat Commun 12:3899.

38. Flanagan CA, Schnieders EA, Emerick AW, Kunisawa R, Admon A, Thorner J. 1993. Phosphatidylinositol 4-kinase: gene structure and requirement for yeast cell viability. Science 262:1444-8.

39. Garcia-Bustos JF, Marini F, Stevenson I, Frei C, Hall MN. 1994. PIK1, an essential phosphatidylinositol 4-kinase associated with the yeast nucleus. EMBO J 13:235261.

40. Kondoh O, Tachibana Y, Ohya Y, Arisawa M, Watanabe T. 1997. Cloning of the RHO1 gene from Candida albicans and its regulation of beta-1,3-glucan synthesis. J Bacteriol 179:7734-41.

41. Tani M, Kuge O. 2014. Involvement of Sac1 phosphoinositide phosphatase in the metabolism of phosphatidylserine in the yeast Saccharomyces cerevisiae. Yeast $31: 145-58$.

42. Chung J, Torta F, Masai K, Lucast L, Czapla H, Tanner LB, Narayanaswamy P, Wenk MR, Nakatsu F, De Camilli P. 2015. PI4P/phosphatidylserine countertransport at ORP5- and ORP8-mediated ER-plasma membrane contacts. Science 349:428-32.

43. Zewe JP, Wills RC, Sangappa S, Goulden BD, Hammond GR. 2018. SAC1 degrades its lipid substrate PtdIns4P in the endoplasmic reticulum to maintain a steep chemical gradient with donor membranes. Elife 7.

44. Omnus DJ, Cadou A, Thomas FB, Bader JM, Soh N, Chung GHC, Vaughan AN, Stefan CJ. 2020. A heat-sensitive Osh protein controls PI4P polarity. BMC Biol $18: 28$.

45. Chung J, Nakatsu F, Baskin JM, De Camilli P. 2015. Plasticity of PI4KIIIalpha interactions at the plasma membrane. EMBO Rep 16:312-20. 
46. Baskin JM, Wu X, Christiano R, Oh MS, Schauder CM, Gazzerro E, Messa M, Baldassari S, Assereto S, Biancheri R, Zara F, Minetti C, Raimondi A, Simons M, Walther TC, Reinisch KM, De Camilli P. 2016. The leukodystrophy protein FAM126A (hyccin) regulates PtdIns(4)P synthesis at the plasma membrane. Nat Cell Biol 18:132-8.

47. Hasim S, Allison DP, Retterer ST, Hopke A, Wheeler RT, Doktycz MJ, Reynolds TB. 2017. beta-(1,3)-Glucan Unmasking in Some Candida albicans Mutants Correlates with Increases in Cell Wall Surface Roughness and Decreases in Cell Wall Elasticity. Infect Immun 85.

48. Fernandes CM, Goldman GH, Del Poeta M. 2018. Biological Roles Played by Sphingolipids in Dimorphic and Filamentous Fungi. mBio 9.

49. Mor V, Rella A, Farnoud AM, Singh A, Munshi M, Bryan A, Naseem S, Konopka JB, Ojima I, Bullesbach E, Ashbaugh A, Linke MJ, Cushion M, Collins M, Ananthula HK, Sallans L, Desai PB, Wiederhold NP, Fothergill AW, Kirkpatrick WR, Patterson T, Wong LH, Sinha S, Giaever G, Nislow C, Flaherty P, Pan X, Cesar GV, de Melo Tavares P, Frases S, Miranda K, Rodrigues ML, Luberto C, Nimrichter L, Del Poeta M. 2015. Identification of a New Class of Antifungals Targeting the Synthesis of Fungal Sphingolipids. mBio 6:e0647.

50. Munshi MA, Gardin JM, Singh A, Luberto C, Rieger R, Bouklas T, Fries BC, Del Poeta M. 2018. The Role of Ceramide Synthases in the Pathogenicity of Cryptococcus neoformans. Cell Rep 22:1392-1400.

51. Noble SM, French S, Kohn LA, Chen V, Johnson AD. 2010. Systematic screens of a Candida albicans homozygous deletion library decouple morphogenetic switching and pathogenicity. Nat Genet 42:590-8. 
52. Ram AF, Klis FM. 2006. Identification of fungal cell wall mutants using susceptibility assays based on Calcofluor white and Congo red. Nat Protoc 1:22536.

53. Wilson RB, Davis D, Mitchell AP. 1999. Rapid hypothesis testing with Candida albicans through gene disruption with short homology regions. J Bacteriol 181:186874.

54. Schaub Y, Dunkler A, Walther A, Wendland J. 2006. New pFA-cassettes for PCRbased gene manipulation in Candida albicans. J Basic Microbiol 46:416-29.

55. Milne SW, Cheetham J, Lloyd D, Aves S, Bates S. 2011. Cassettes for PCR-mediated gene tagging in Candida albicans utilizing nourseothricin resistance. Yeast 28:83341.

56. Care RS, Trevethick J, Binley KM, Sudbery PE. 1999. The MET3 promoter: a new tool for Candida albicans molecular genetics. Mol Microbiol 34:792-8.

57. Silva PM, Puerner C, Seminara A, Bassilana M, Arkowitz RA. 2019. Secretory Vesicle Clustering in Fungal Filamentous Cells Does Not Require Directional Growth. Cell Rep 28:2231-2245 e5.

58. Puerner C, Kukhaleishvili N, Thomson D, Schaub S, Noblin X, Seminara A, Bassilana M, Arkowitz RA. 2020. Mechanical force-induced morphology changes in a human fungal pathogen. BMC Biol 18:122.

59. Sanchez AA, Johnston DA, Myers C, Edwards JE, Jr., Mitchell AP, Filler SG. 2004. Relationship between Candida albicans virulence during experimental hematogenously disseminated infection and endothelial cell damage in vitro. Infect Immun 72:598-601.

60. Solis NV, Filler SG. 2012. Mouse model of oropharyngeal candidiasis. Nat Protoc 7:637-42. 
61. Weiner A, Orange F, Lacas-Gervais S, Rechav K, Ghugtyal V, Bassilana M, Arkowitz RA. 2019. On-site secretory vesicle delivery drives filamentous growth in the fungal pathogen Candida albicans. Cell Microbiol 21:e12963. 
A
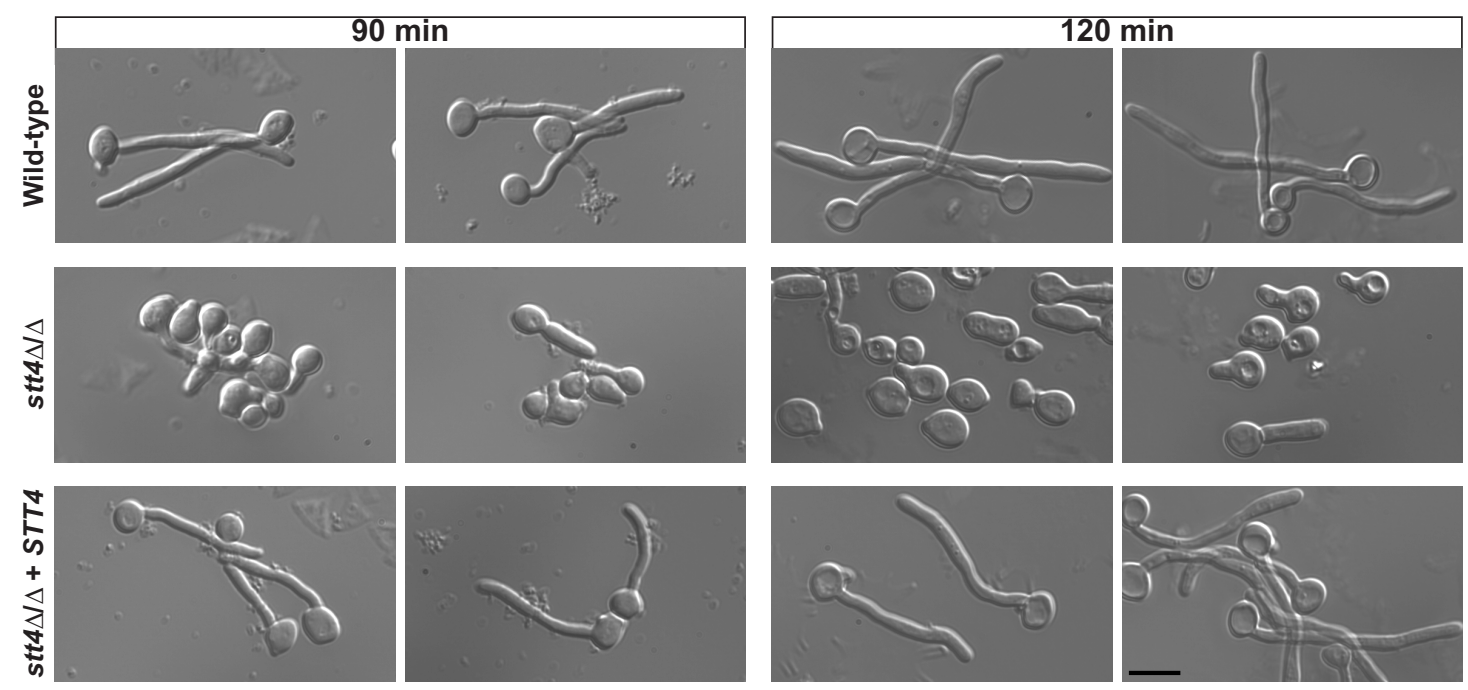

B

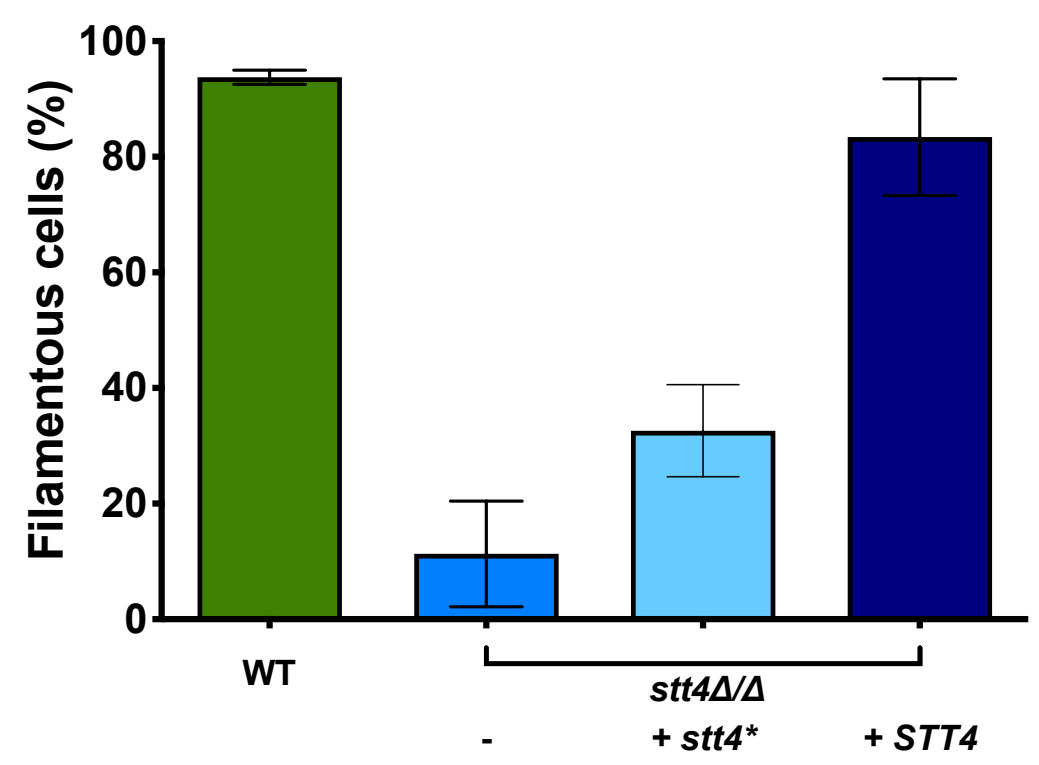

\section{Strain}

C

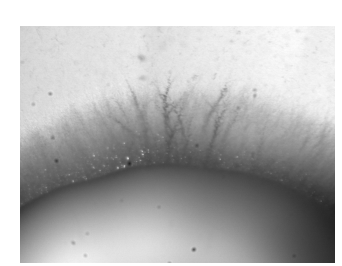

Wild-type

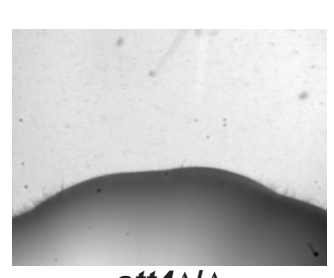

$\operatorname{stt} 4 \Delta \Delta$

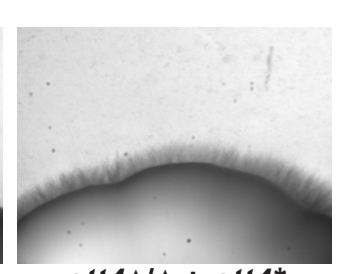

stt4 $\Delta \Delta+$ stt4* $^{*}$

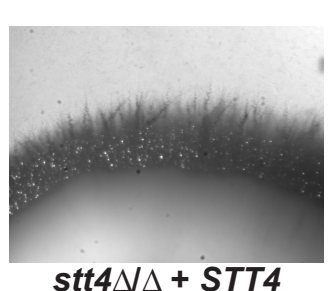

$\operatorname{stt4} \Delta \Delta+$ STT4

Figure 1. The PI-4-kinase Stt4 is required for filamentous growth. A) Indicated strains (wild-type, PY4861; stt4 $/ \Delta$, PY5111; stt4 $/ \Delta+S T T 4$, PY5131) were incubated with serum at $37^{\circ} \mathrm{C}$ for 90 and $120 \mathrm{~min}$. B) Percentage of filamentous cells were determined from three independent experiments. ( $n \geq 120$ in each) with strains indicated above in addition to $s t t 4 \Delta / \Delta+s t t 4 *$ encoding Stt4[G1810D], PY5757. Cells were considered filametous if germ tubes were twice length of the mother cell or longer, error bars indicate SD. C) Stt4 is required for invasive filamentous growth. Indicated strains were incubated for 4 days at $30^{\circ} \mathrm{C}$ on serum agar plates. Similar results were observed in three independent experiments. 
A
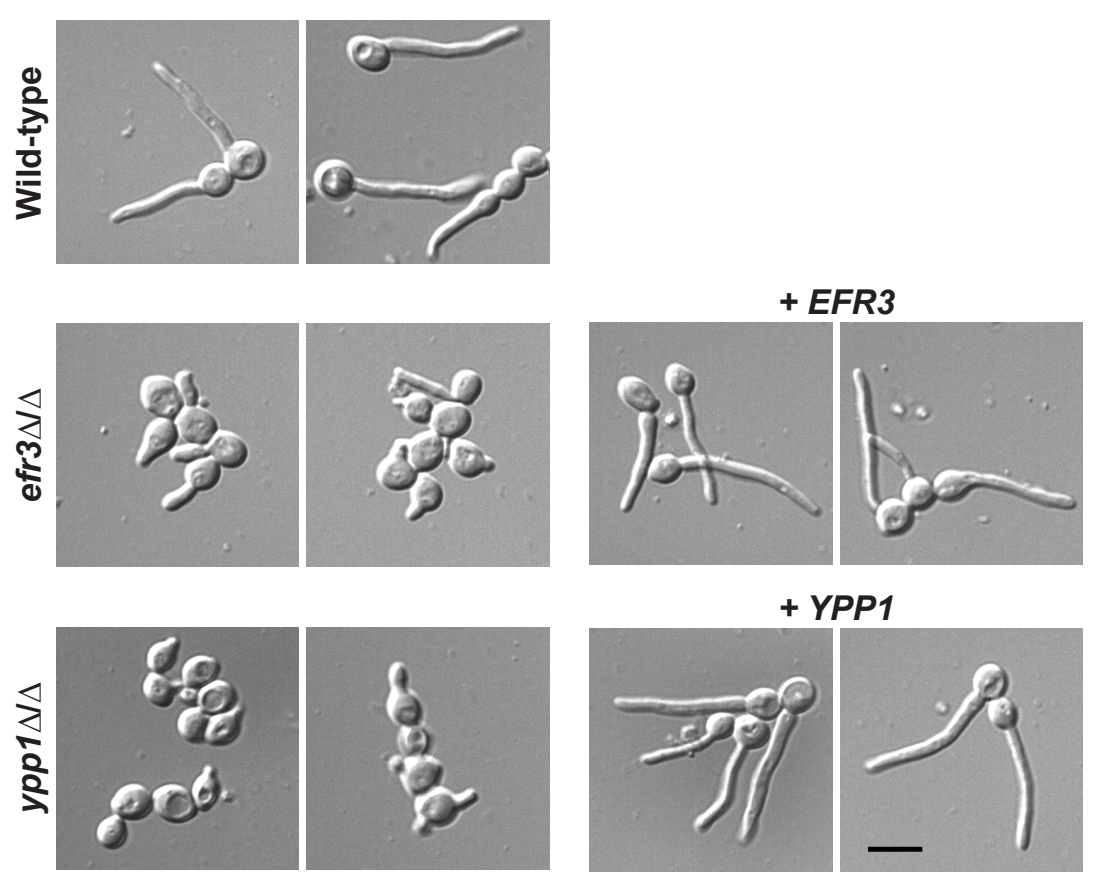

B

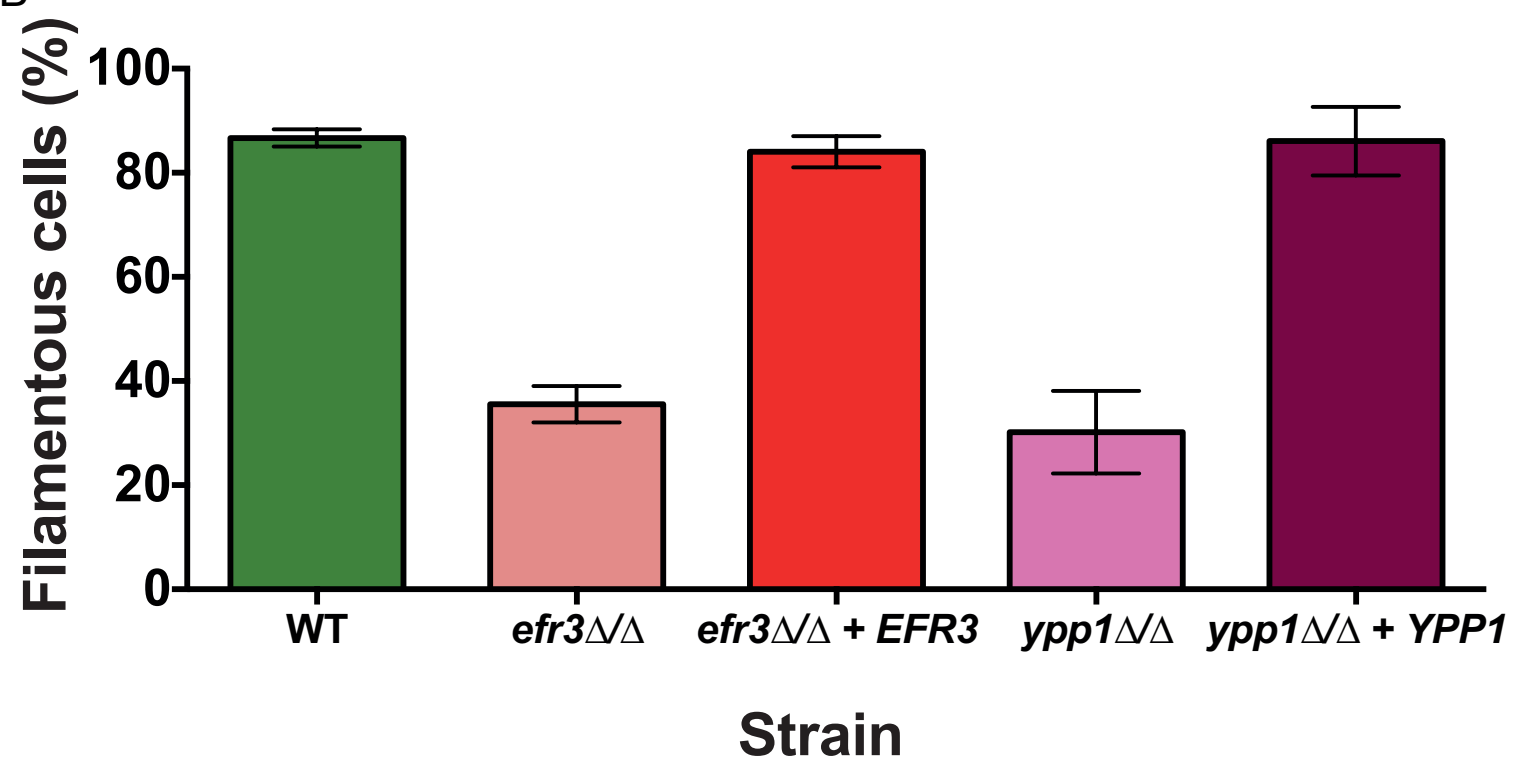

C

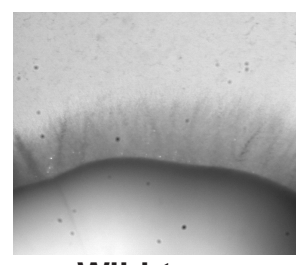

Wild-type

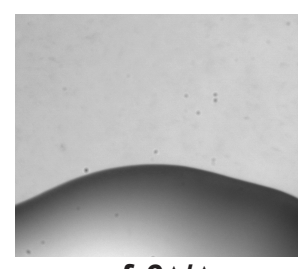

efr $3 \Delta / \Delta$

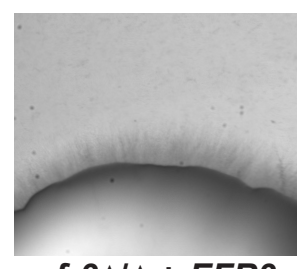

efr3 $\Delta / \Delta+E F R 3$

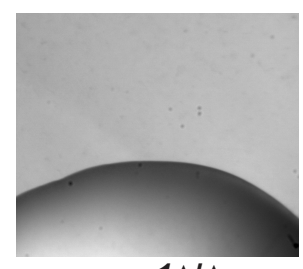

$\operatorname{ypp} 1 \Delta / \Delta$

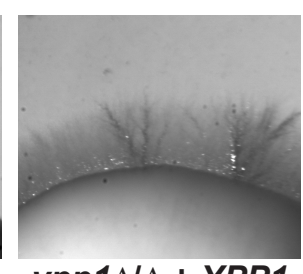

ypp1 $1 \Delta \Delta+Y P P 1$

Figure 2. Efr3 and Ypp1 are required for filamentous growth. A) Indicated strains (wild-type, PY4861; efr $3 \Delta / \Delta$, PY4036; efr $3 \Delta / \Delta+E F R 3$, PY4039; ypp $1 \Delta / \Delta$, PY4033; ypp $1 \Delta / \Delta+Y P P 1$, PY4040) were induced with serum at $37^{\circ} \mathrm{C}$ for $90 \mathrm{~min}$. B) Percentage of filamentous cells was determined from three independent experiments ( $n \geq 120$ in each). Error bars indicate SD. C) Efr3 and Ypp1 are required for invasive filamentous growth. Indicated strains were incubated for 4 days at $30^{\circ} \mathrm{C}$ on serum agar plates. Similar results were observed in three independent experiments. 


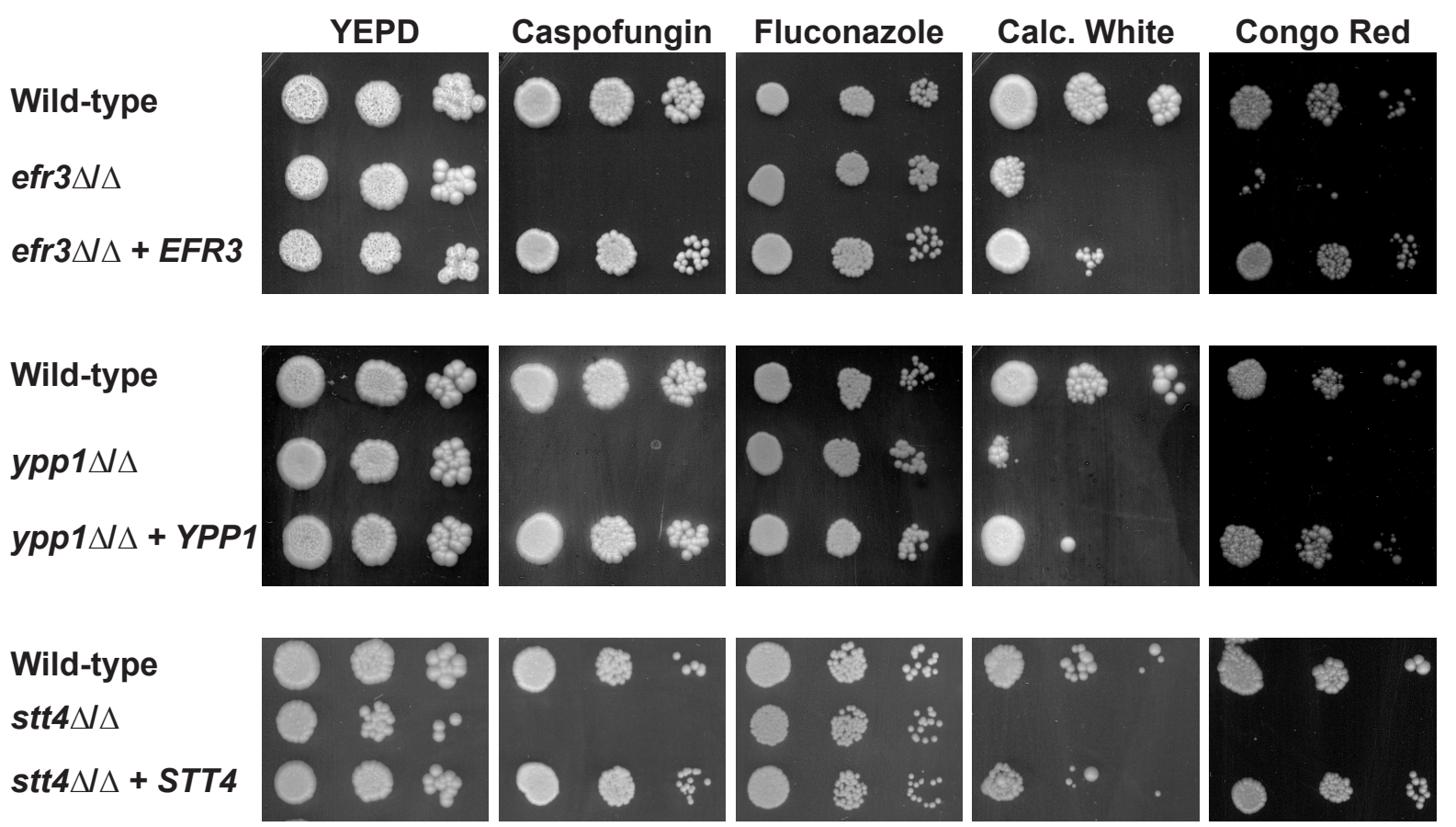

Figure 3. Plasma membrane PI(4)P is important for cell wall integrity. Indicated strains (wild-type, PY4861; efr $3 \Delta / \Delta$, PY4036; efr $3 \Delta / \Delta+E F R 3$, PY4039; ypp $1 \Delta / \Delta$,

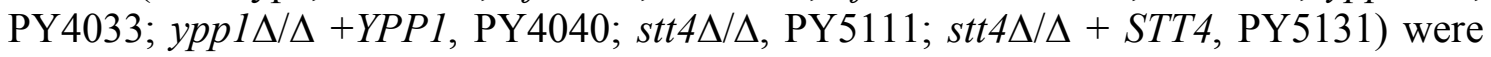
incubated on YEPD with or without caspofungin $(125 \mathrm{ng} / \mathrm{mL})$, fluconazole $(10 \mu \mathrm{g} / \mathrm{mL})$, Calcofluor White $(25 \mu \mathrm{g} / \mathrm{mL})$ or Congo Red $(400 \mu \mathrm{g} / \mathrm{mL})$ for 3 days at $30^{\circ} \mathrm{C}$. Similar results were observed in two independent experiments. 
A
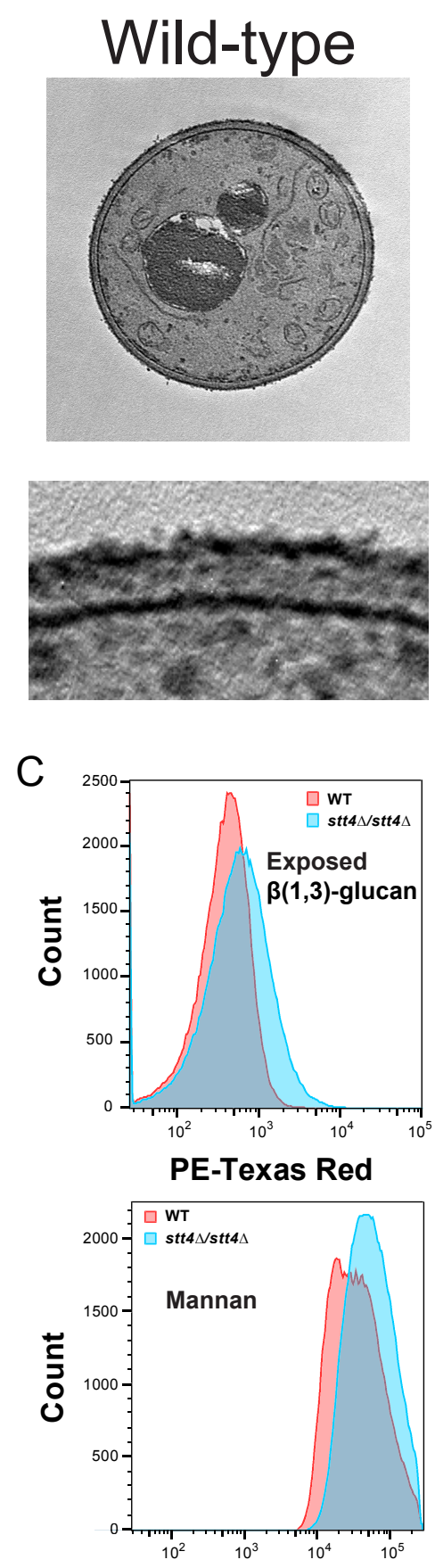

PE-Texas Red

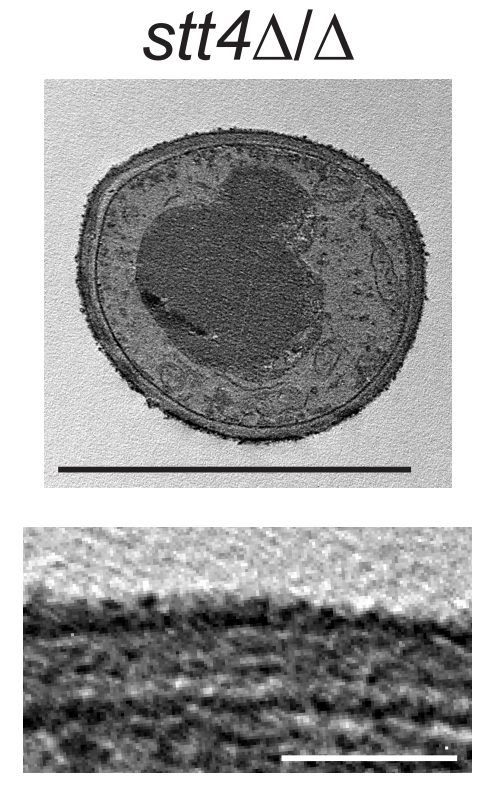

B
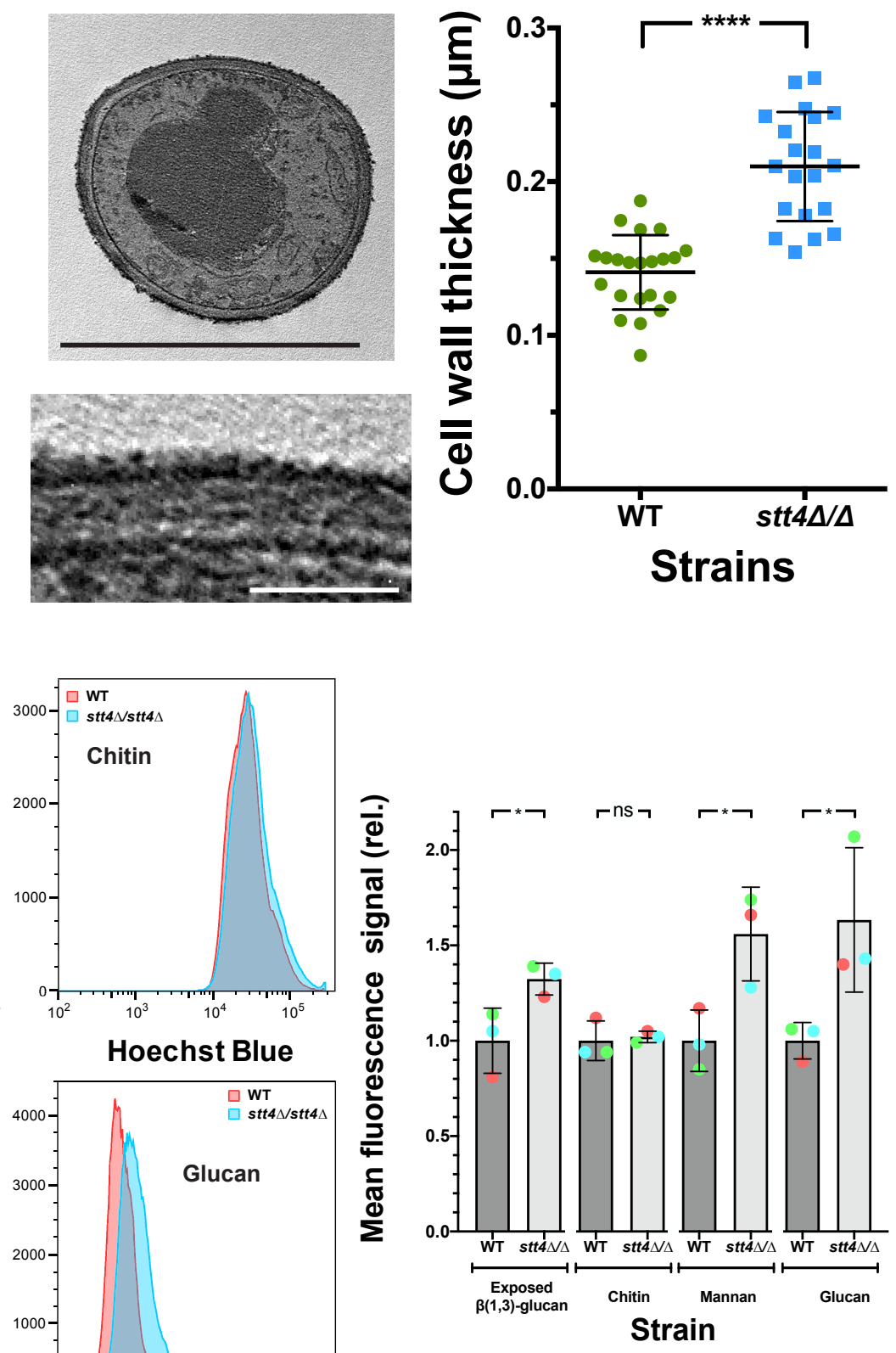

Figure 4. Stt4 deletion mutant has a thicker cell wall with increased mannan, glucan and exposed $\boldsymbol{\beta}(\mathbf{1}, \mathbf{3})$-glucan. A) Transmission electron micrographs of the indicated strains (wild-type, PY4861; stt4 $\Delta / \Delta$, PY5111) (upper) with zoom in on the cell wall. Scale bar is $5 \mu \mathrm{m}$ (upper panel) and $1 \mu \mathrm{m}$ (lower panel). B) Quantitation of cell wall thickness from EM micrographs. C) The stt4 mutant has increased exposure of $\beta(1,3)$-glucan together with increased levels of mannan and glucan. Flow cytometry analyses of indicated cells (wild-type, PY4861; stt4 $\Delta / \Delta$, PY5111) labeled with anti- $\beta(1,3)$-glucan antibodies and a fluorescently labeled secondary antibody, calcofluor white, fluorescently labeled concanavalin A and aniline blue. Flow cytometry profiles from one biological replicate $\left(10^{5}\right.$ gated events; left $)$ and means from three biological replicates normalized to each wild-type mean, respectively (right). Bars indicate standard deviations, $*$ is $\mathrm{p}<$ $0.05, * * * *$ is $p<0.0001$ and $\mathrm{ns}$ is not significant. 
A
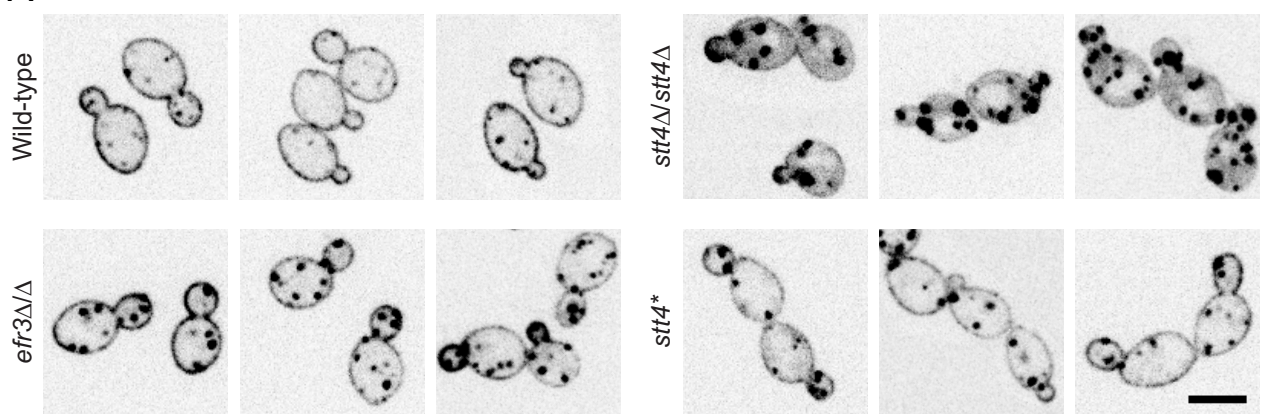
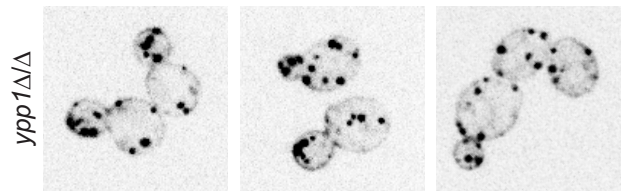

B

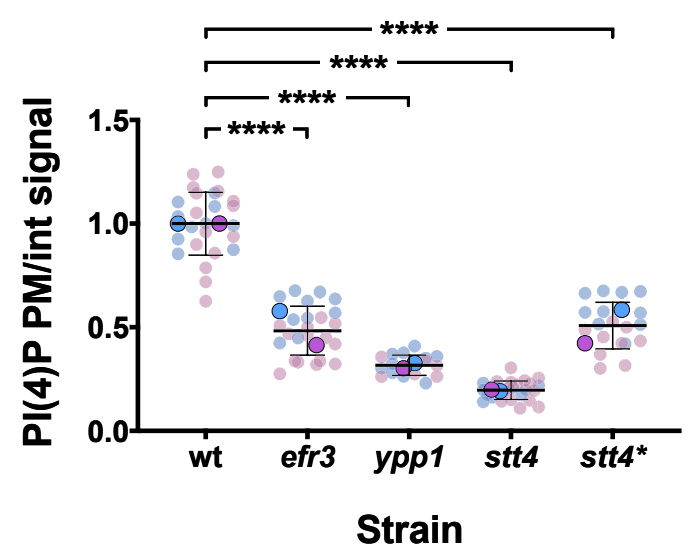

C

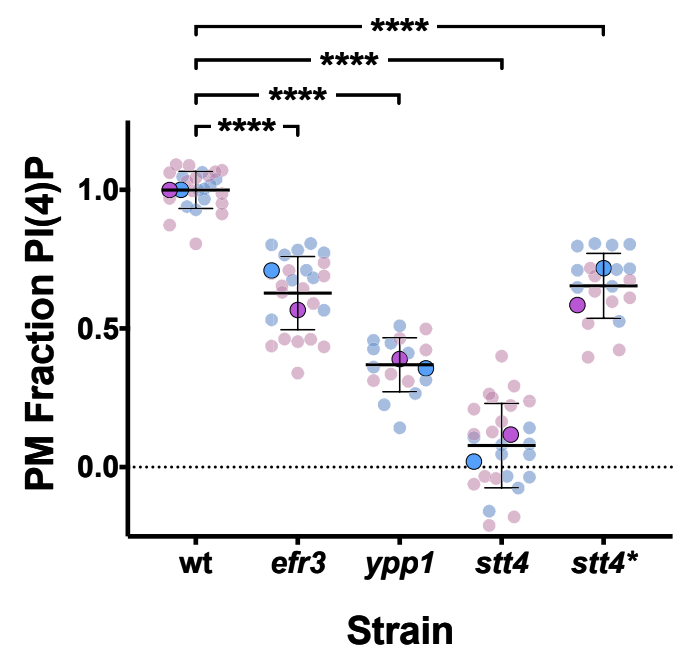

Figure 5. Efr3, Ypp1 and Stt4 are critical for plasma membrane PI(4)P. A) Indicated strains expressing the plasma membrane PI(4)P reporter, GFP-Osh2 ${ }^{\mathrm{PH}}-\mathrm{Osh}_{2}{ }^{\mathrm{PH}}$-GFP (wild-type, PY2626; efr $3 \Delta / \Delta$, PY4947; ypp $1 \Delta / \Delta$, PY3950; stt4 $\Delta / \Delta$, PY5169; stt4 $\Delta / \Delta+$ stt4*, PY5838) were imaged and central z-sections of representative cells are shown with inverted look up table (LUT). B - C) Quantitation of plasma membrane and internal signals reveal little to no plasma membrane PI(4)P the stt4 mutant. The ratio of plasma membrane to internal signal (normalized to the wild-type) and the relative plasma membrane signal (normalized plasma membrane/total signal) is shown. Quantitation of plasma membrane and internal signals was carried out excluding Golgi cisternae. For the wild-type, the mean ratio plasma membrane to internal signal was 3.8 and the ratio plasma membrane divided by total signal was 0.8 . We are able to detect $\sim 1.5 \%$ of wild-type plasma membrane PI(4)P levels. Smaller symbols are values from two experiments (6-15 cells per experiment), larger symbols are mean of each experiment with bars indicating overall means and standard deviations. ${ }^{* * * *}$ is $p<0.0001$ 
A

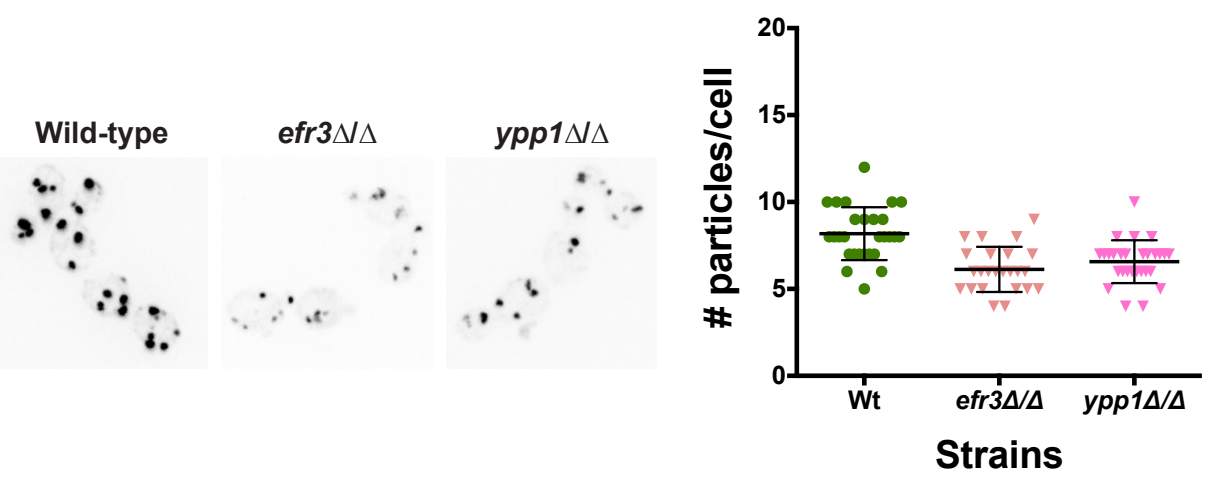

B

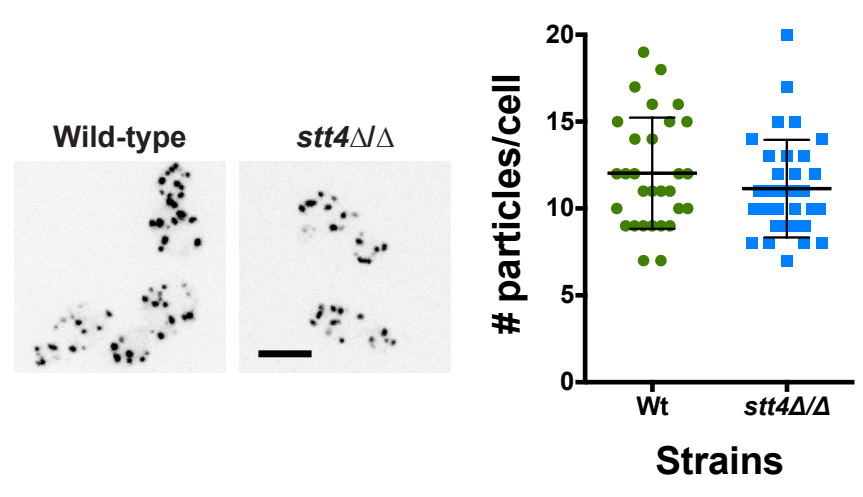

Figure 6. The number of Golgi cisternae is not affected by a decrease in plasma membrane PI(4)P. A-B) Indicated strains expressing Golgi PI(4)P reporter, FAPP1-GFP (wild-type, PY2578; efr3 $\Delta / \Delta$, PY3933; ypp $1 \Delta / \Delta$, PY3951; stt4 $/ \Delta$, PY5552) were imaged and maximum projections of representative cells are shown with inverted LUT (left). Quantitation of the number of Golgi cisternae per cell (right) in the indicated strains $(n=24-34$ cells per strain). 
A

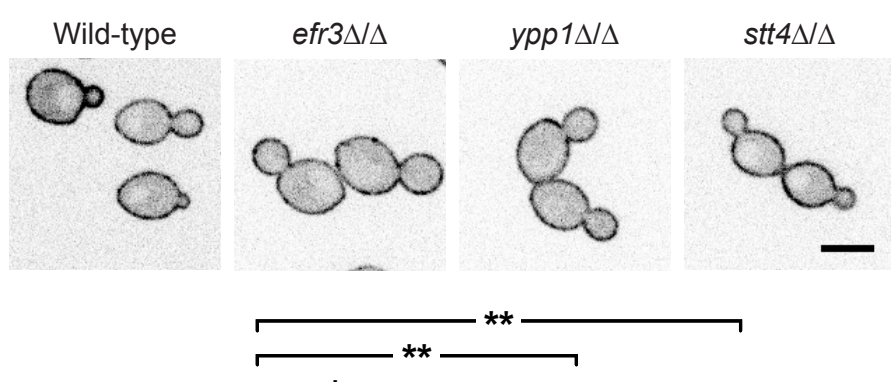

B

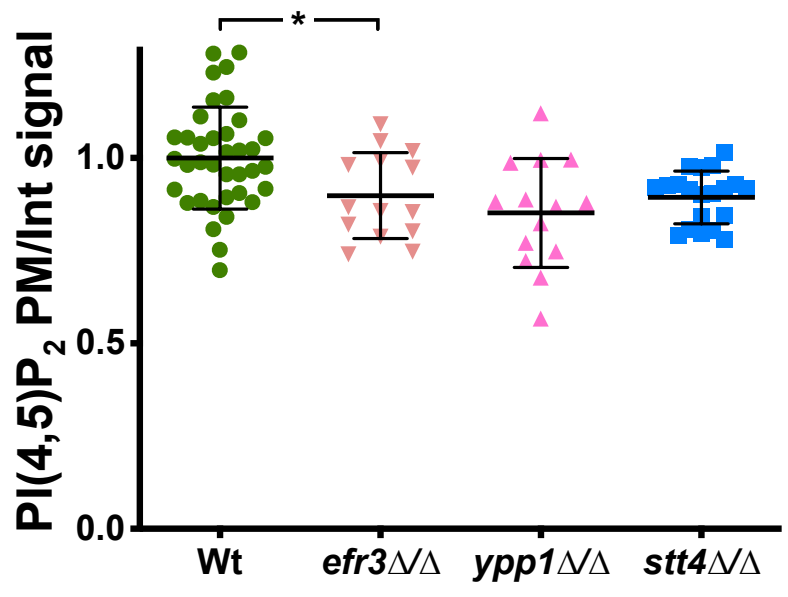

Strain

C

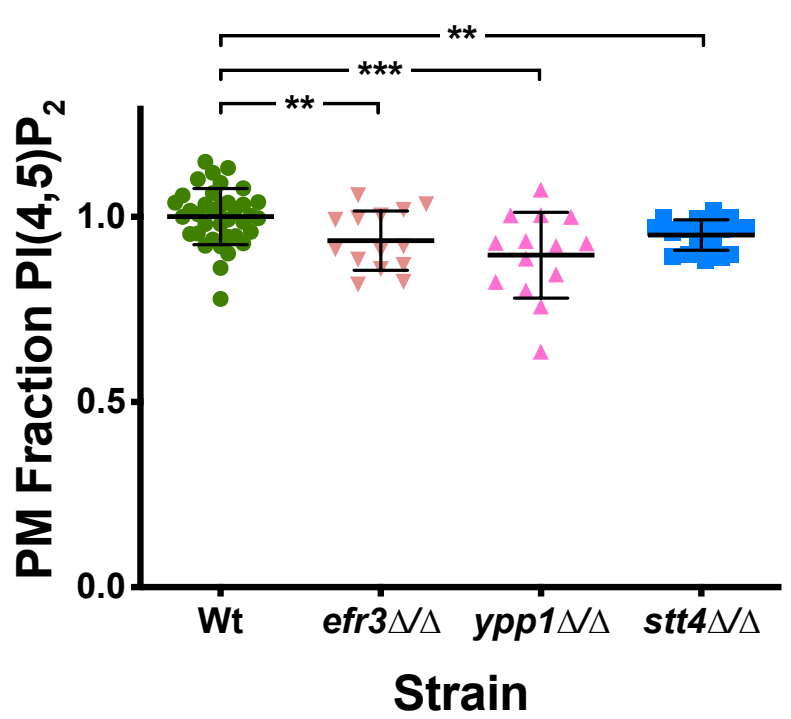

Figure 7. Plasma membrane $\mathrm{PI}(4,5) \mathrm{P}_{2}$ is not substantially affected by a decrease in PI(4)P. A) Indicated strains expressing PI(4,5) $\mathrm{P}_{2}$ reporter, GFP-PH ${ }^{\mathrm{Plc} \delta}-\mathrm{PH}^{\mathrm{Plc} \delta}-\mathrm{GFP}$ wildtype, PY1206; efr $3 \Delta / \Delta$, PY3935; ypp $1 \Delta / \Delta$, PY3958; stt4 $\Delta / \Delta$, PY555) were imaged and central z-sections of representative cells are shown with inverted LUT. B-C) Quantitation of plasma membrane and internal signals reveals that plasma membrane $\mathrm{PI}(4,5) \mathrm{P}_{2}$ is largely unaffected in the absence of Efr3, Ypp1 and Stt4. The ratio of plasma membrane to internal signal and the relative plasma membrane signal determined as in Figure 5B, $5 \mathrm{C}(n=15-20$ cells; 2 experiments for WT). For the wild-type, the mean ratio plasma membrane to internal signal was 2.8 and the ratio plasma membrane divided by total signal was $0.7 . *<0.02, * *$ is $\mathrm{p}<0.01$ and $* * *$ is $p<0.0005$. 
A

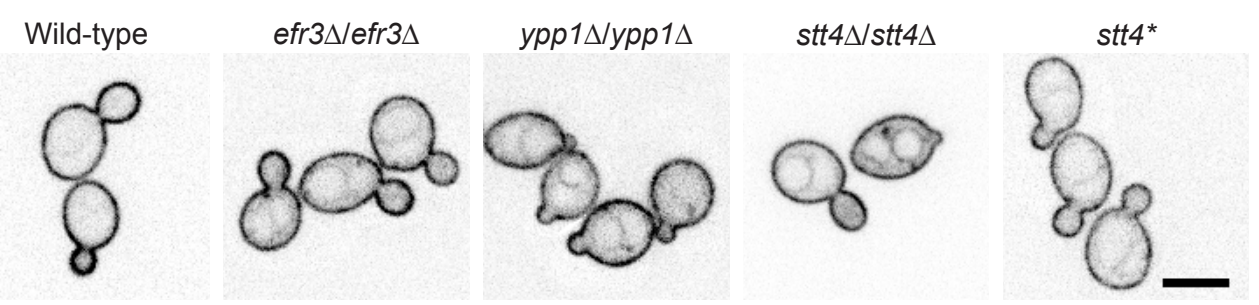

B

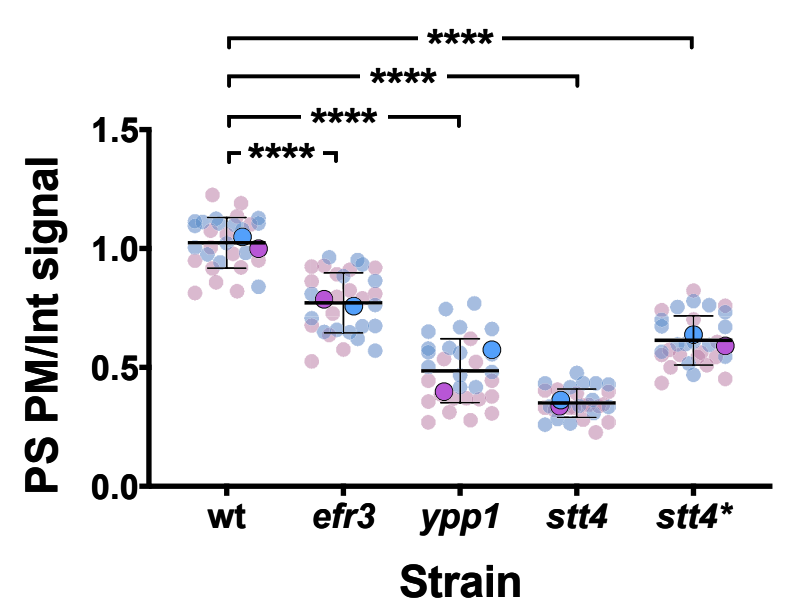

C

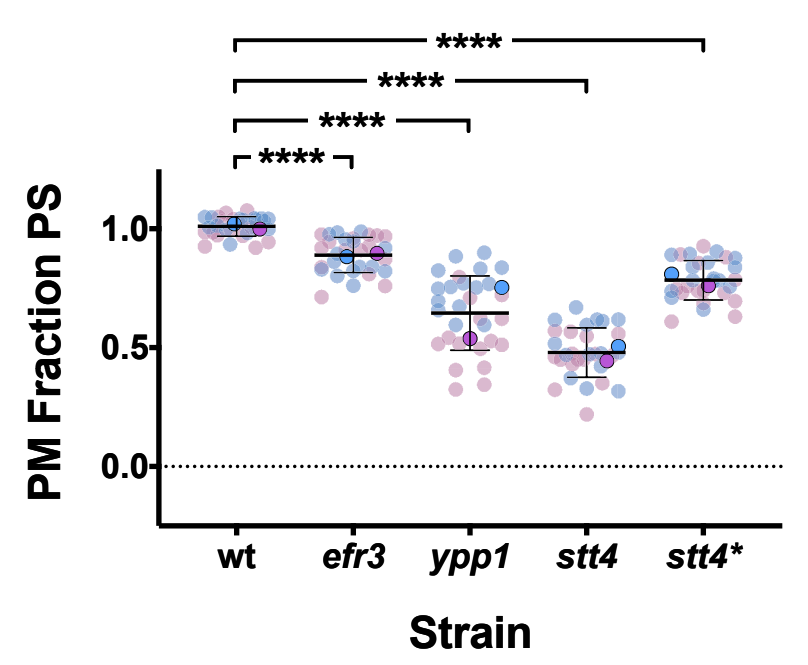

Figure 8. A reduction in plasma membrane PI(4)P results in an increase in PS at the ER. A) Indicated strains expressing PS reporter GFP-LactC2 (wild-type, PY3239; efr $3 \Delta / \Delta$, PY4124; ypp $1 \Delta / \Delta$, PY4131; stt4 $/ \Delta$, PY5174; stt4 $\Delta / \Delta+$ stt4*, PY5903) were imaged and central z-sections of representative cells are shown with inverted LUT. B-C) Quantitation of plasma membrane and internal signals reveals a progressive decrease in plasma membrane PS in efr 3, yppl and stt4 strains. The ratio of plasma membrane to internal signal and the relative plasma membrane signal determined as in Figure 5B, 5C. For the wild-type, the mean ratio plasma membrane to internal signal was 4.5 and the ratio plasma membrane divided by total signal was 0.8 . Smaller symbols are values from two experiments ( $n=15$ cells each), larger symbols are mean of each experiment with bars indicating overall means and standard deviations. ${ }^{* * * *}$ is $p<0.0001$ 


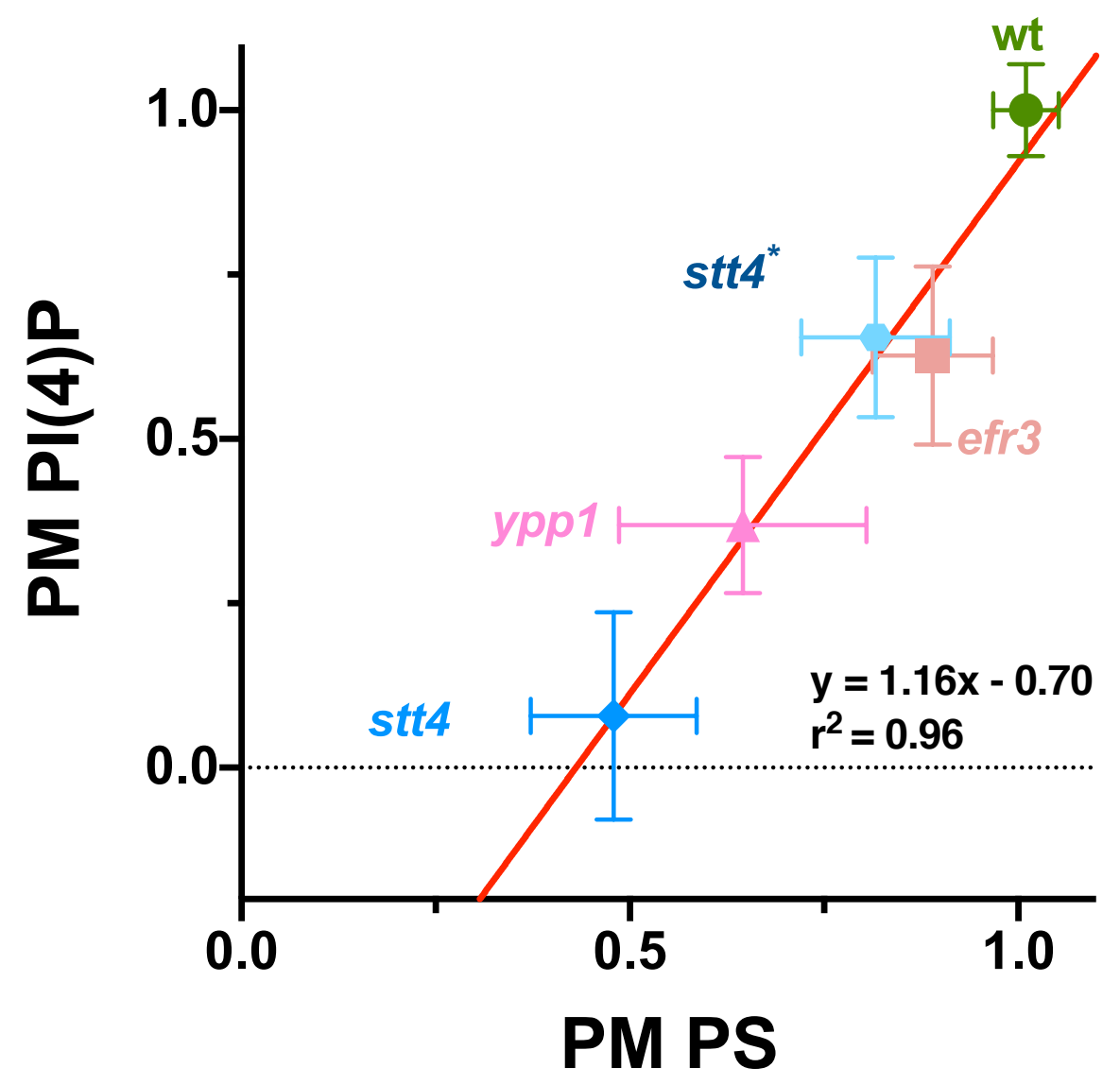

Figure 9. Plasma membrane PS is proportional to PI(4)P levels. Average levels of plasma membrane PS and PI(4)P in indicated strains were normalized to 1 in the wildtype. Linear curve fit: $y=1.316 x-0.70 ; \mathrm{r}^{2}=0.96$. Bars are standard deviation with $n$ $=16-40$ cells for each determination. 
bioRxiv preprint doi: https://doi.org/10.1101/2021.12.31.474627; this version posted January 1, 2022. The copyright holder for this preprint (which was not certified by peer review) is the author/funder. All rights reserved. No reuse allowed without permission.

A
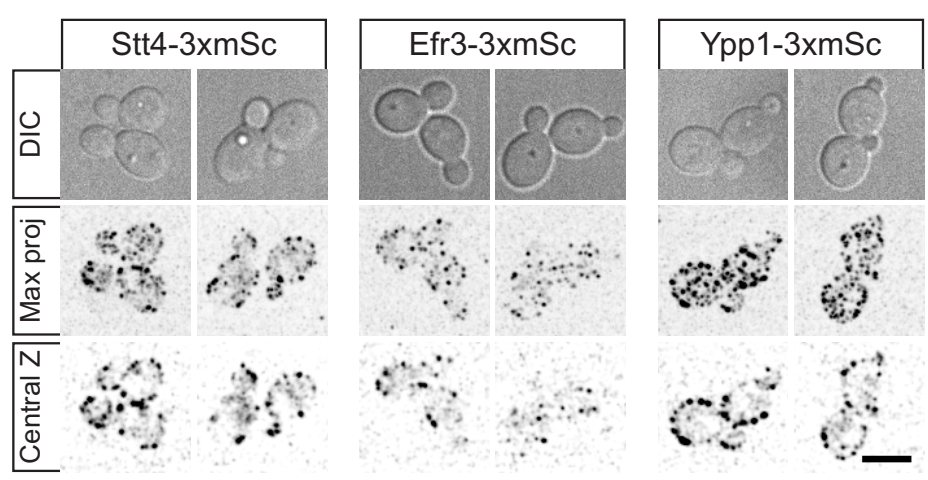

B

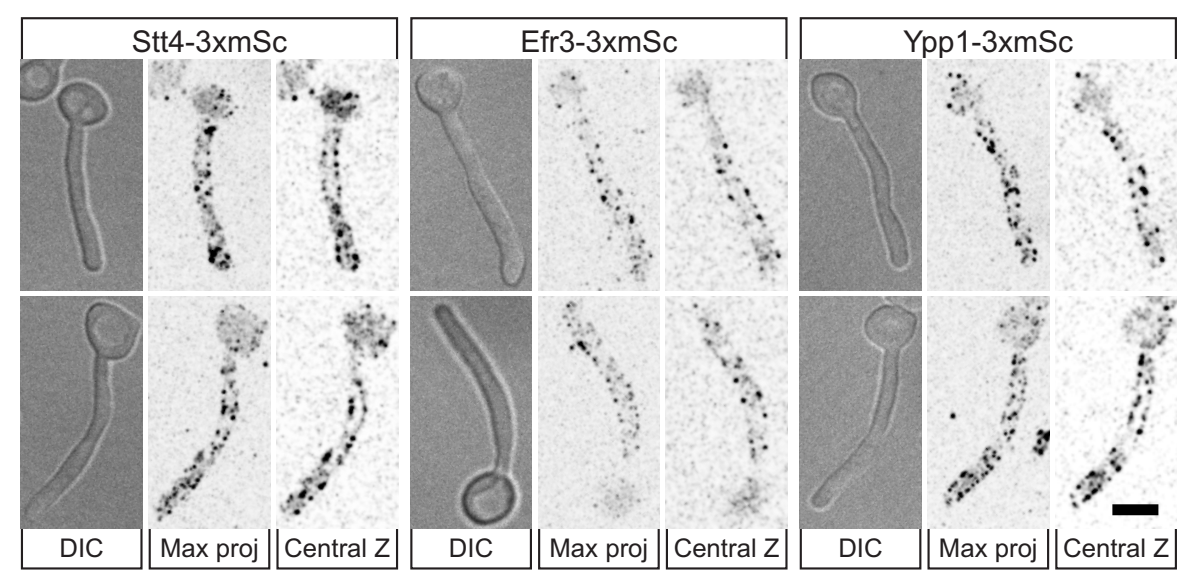

C
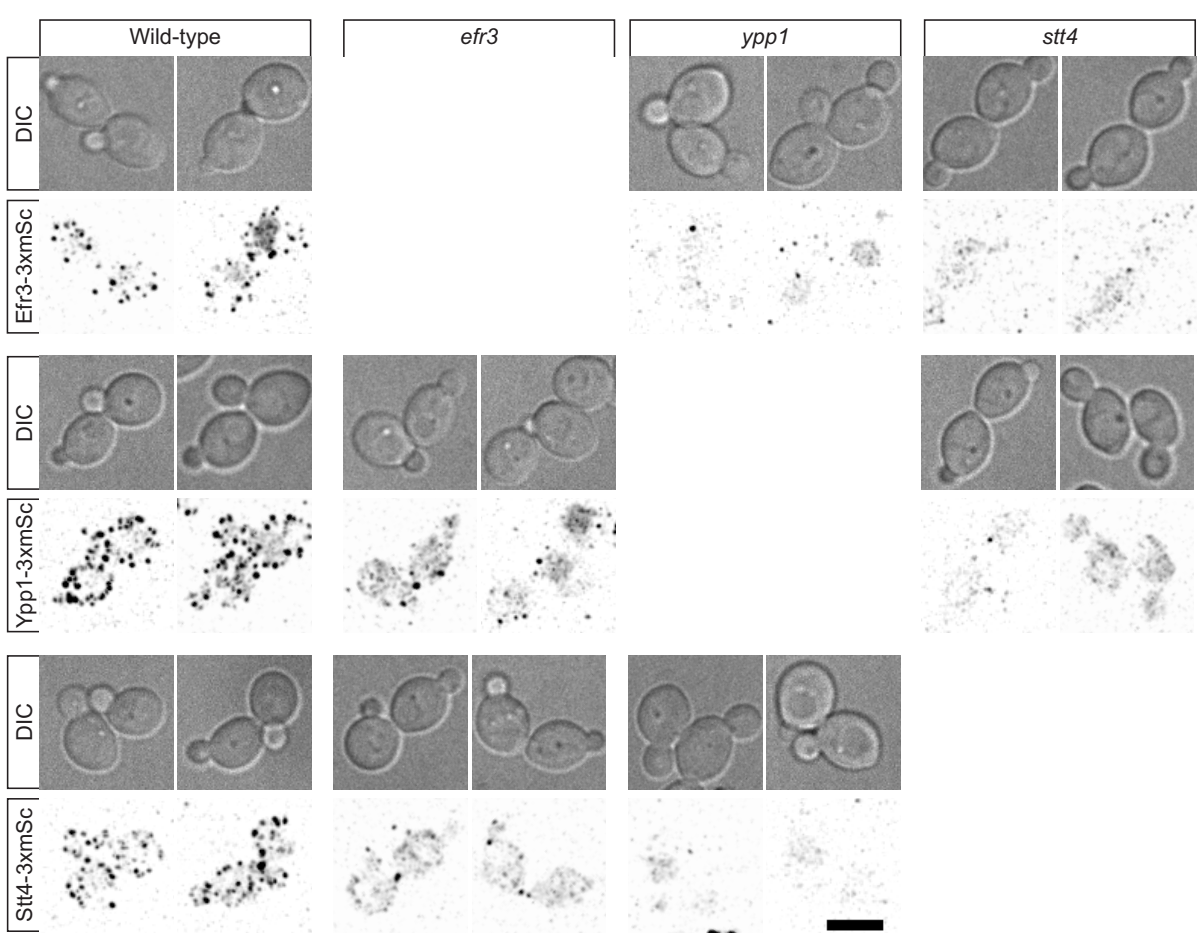

Figure 10. Efr3, Ypp1 and Stt4 localize as cortical patches, with Ypp1 and Stt4 critical for each other's localization. A-B) Strains expressing indicated 3xmScarlet fusions (Stt4-3xmSc, PY6193; Efr3-3xmSc, PY6197; Ypp1-3xmSc, PY6195) were imaged during budding (A) and hyphal (B) growth. DIC images, central z-sections and maximum projections of $17 \times 0.5 \mu \mathrm{m}$ z-sections are shown. C) Indicated strains (WT, PY6197, PY6195, PY6193; efr3, PY6136, PY6142; ypp1, PY6138, PY6144; stt4, PY6140, PY6134) strains expressing respective 3xmScarlet fusions were imaged during budding growth and maximum projections of $17 \times 0.5 \mu \mathrm{m}$ z-sections are shown. 


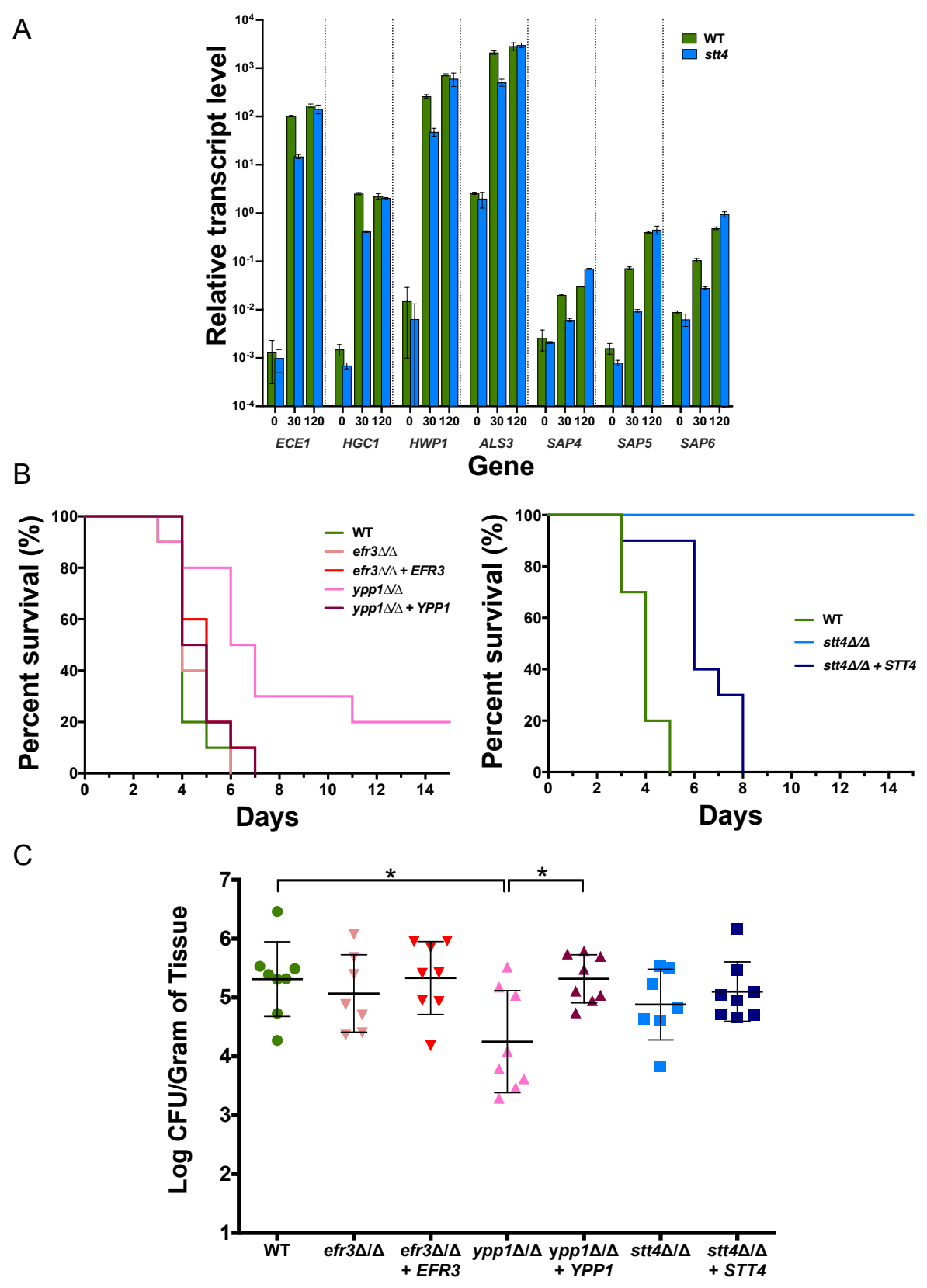

Figure 11. Plasma membrane $\mathrm{PI}(4) \mathrm{P}$ is specifically required for hematogenously disseminated candidiasis. A) Hyphal specific genes are induced in a stt4 deletion mutant. The transcript level of indicated hyphal specific genes was determined in wildtype (PY4861) and stt4 $\Delta / \Delta$ (PY5111) strains at indicated times (min) with serum at $37^{\circ} \mathrm{C}$ by qRT-PCR and normalized to the levels of TDH3 transcript. Means of 3 determinations from an experiment are shown with bars indicating standard deviations. Similar results were observed in two additional biological replicates. B) Stt4 is required for virulence in a murine systemic infection model. Survival of mice $(n=10)$ over time following injection of indicated strains (wild-type, PY4861; efr $3 \Delta / \Delta$, PY4036; efr $3 \Delta / \Delta+E F R 3$, PY4039; ypp $1 \Delta / \Delta$, PY4033; ypp $1 \Delta / \Delta+Y P P 1, \mathrm{PY} 4040 ;$ stt4 $\Delta / \Delta$, PY5111; stt4 $\Delta / \Delta+S T T 4$, PY5131). Similar results were observed in two independent experiments, differences between WT and ypp $1 \Delta / \Delta$ was statistically significant, $p=0.002$ (left panel) and between WT and $s t t 4 \Delta / \Delta$ was statistically significant, $p<0.0001$ (right panel). C) Stt4 and Efr3 are not required for virulence in a murine oropharyngeal infection model. Colony forming units per gram of tongue tissue, 5 days subsequent to oropharyngeal infection $(n=8$ mice per strain) with indicated strains (wild-type, PY4861; efr $3 \Delta / \Delta$, PY4036; efr $3 \Delta / \Delta+E F R 3$, PY4039; ypp $1 \Delta / \Delta$, PY4033; ypp $1 \Delta / \Delta+Y P P 1, \mathrm{PY} 4040 ;$ stt4 $\Delta / \Delta$, PY4414; stt4 $\Delta / \Delta+S T T 4$, PY4433). * is $p<0.05$. 
A
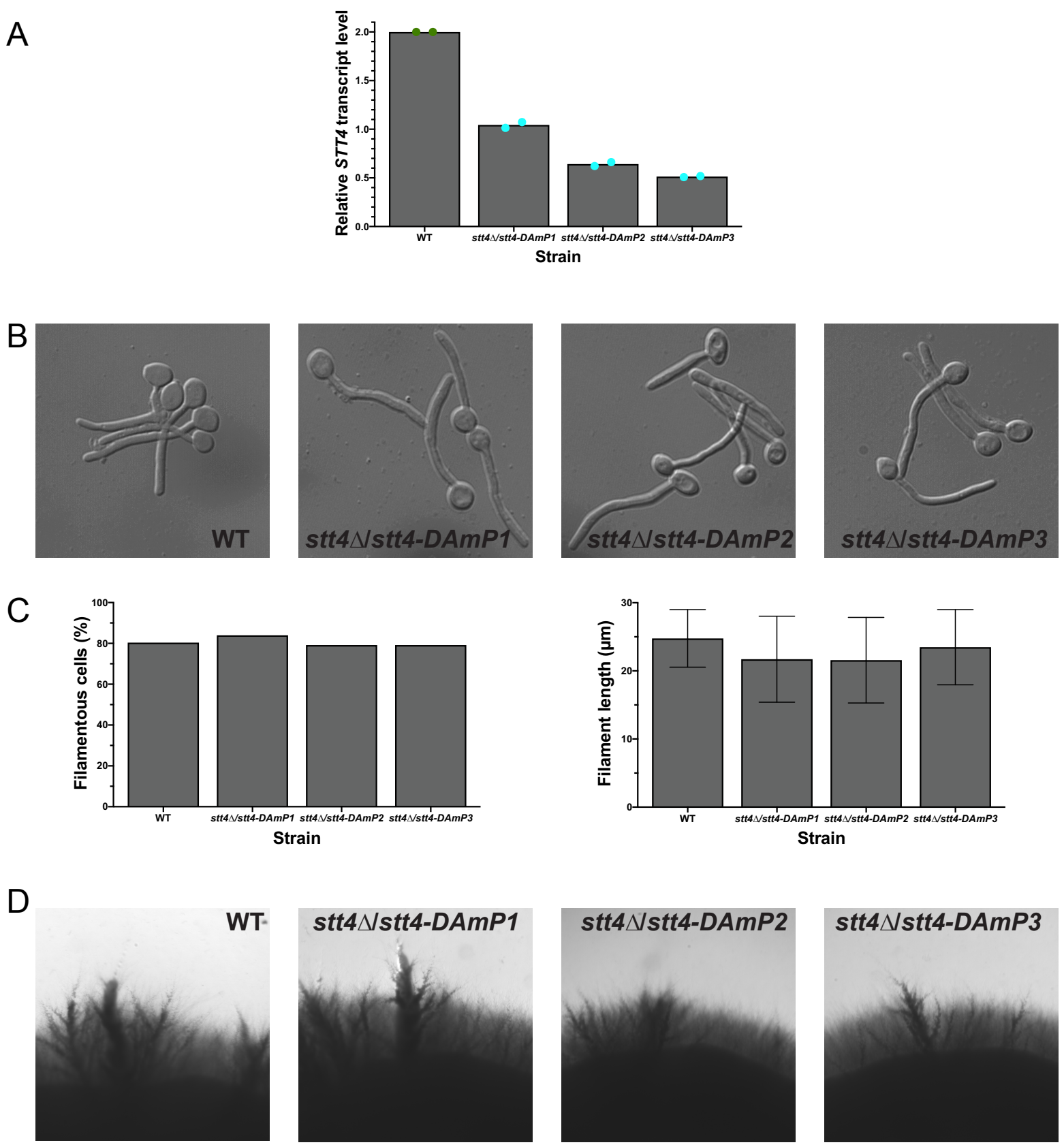

Figure S1. A stt4 decreased abundance by mRNA perturbation mutants undergoes invasive filamentous growth. A) RT-PCR was carried out on indicated strains (wild-type, PY4861; stt4A/stt4-DAmP1-3, PY4339-4341) using CaSTT4TM1 and CaSTT4TM2 primer pairs. Fragments migrated at the expected sizes. Values are the means of determinations with two primer pairs normalized to $A C T 1$. B) Indicated strains were incubated in serum at $37^{\circ} \mathrm{C}$ for $90 \mathrm{~min}$ and images acquired. C) Percentage of filamentous cells (left) ( $n=350$ cells per strain) and hyphal filament lengths (right) were determined ( $n=60$ cells per strain) with error bars indicating SD. D) Indicated strains were incubated for 4 days at $30^{\circ} \mathrm{C}$ on agar serum plates and images acquired. 
bioRxiv preprint doi: https://doi.org/10.1101/2021.12.31.474627; this version posted January 1, 2022. The copyright holder for this preprint (which was not certified by peer review) is the author/funder. All rights reserved. No reuse allowed without permission. S2

A

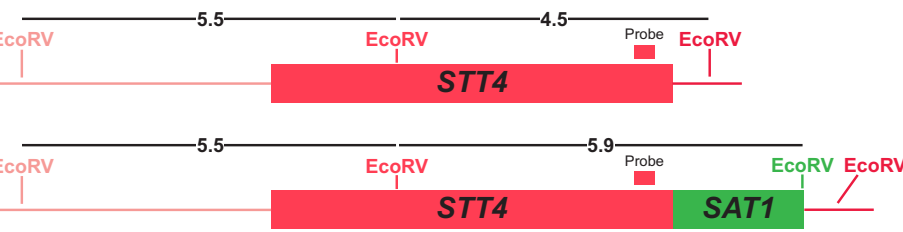

B

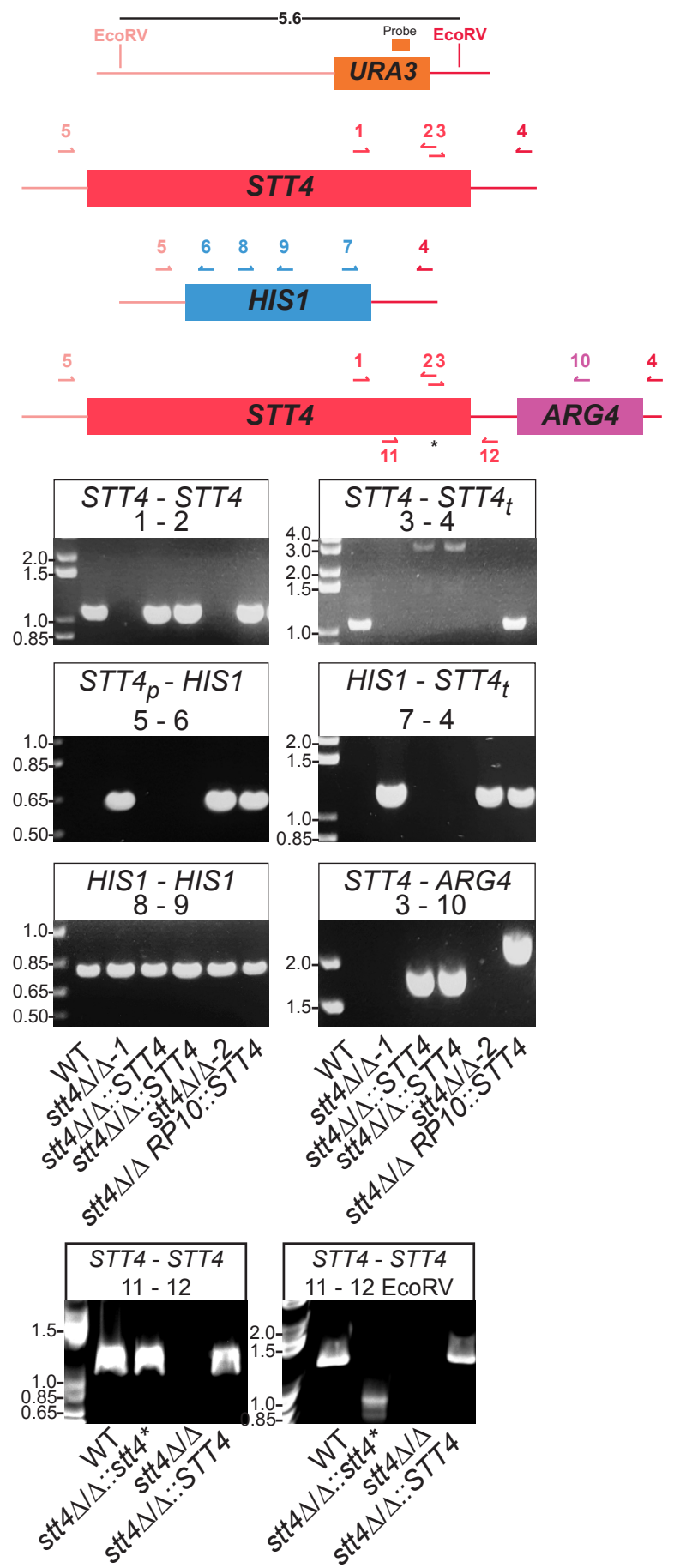

C
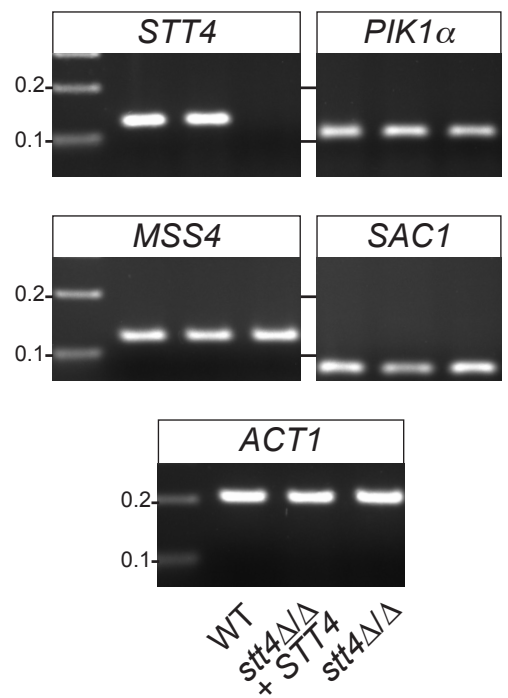

Figure S2. Molecular analyses of stt4 deletion mutants. A) Southern blot analysis of stt4 mutants. Schematic representation of chromosomal restriction sites and probes (STT4 probe, CaStt4p5199 and CaStt4m5543; URA3 probe, CaUra3pXhoI and CaUra3m81) (left) and Southern blot using STT4 and URA3 probes, size in $\mathrm{kB}$, of indicated strains (wild-type, PY4861;

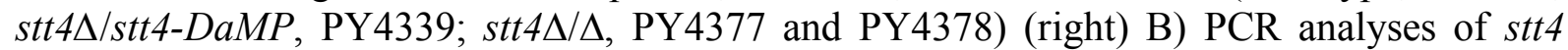
mutants. Schematic representation of STT4 with primers used for strain verification indicated (top) and PCR analyses of indicated strains (wild-type, PY4861; stt4 $\Delta / \Delta-1$, PY5111;

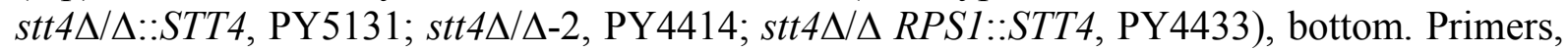
1 CaStt4p4100; 2 CaStt4m4255; 3 CaStt4p5519; 4 CaStt4m6600NotI; 5 CaStt4pup325; 6 CaHIS1pStop1008; 7 CaHIS1mup152; 8 CaHIS1p214; 9 CaHIS1m836; 10 CaARG4m537; 11 
CaStt4p4884; 12 CaStt4m5857. Star indicates position of mutation encoding [G1810D]. C) PIK1, MSS4 and SAC1 mRNA transcripts are not altered in the stt4 mutant. RT-PCR was carried out on indicated strains (wild-type, PY4861; stt4 $\Delta / \Delta+S T T 4$, PY5131; stt4 $\Delta / \Delta$, PY5111) with primers for indicated gene amplifications. Fragments migrated at the expected sizes and ACT1 controls revealed similar amounts of cDNA in each strain. 
bioRxiv preprint doi: https://doi.org/10.1101/2021.12.31.474627; this version posted January 1, 2022. The copyright holder for this preprint (which was not certified by peer review) is the author/funder. All rights reserved. No reuse allowed without permission.

A
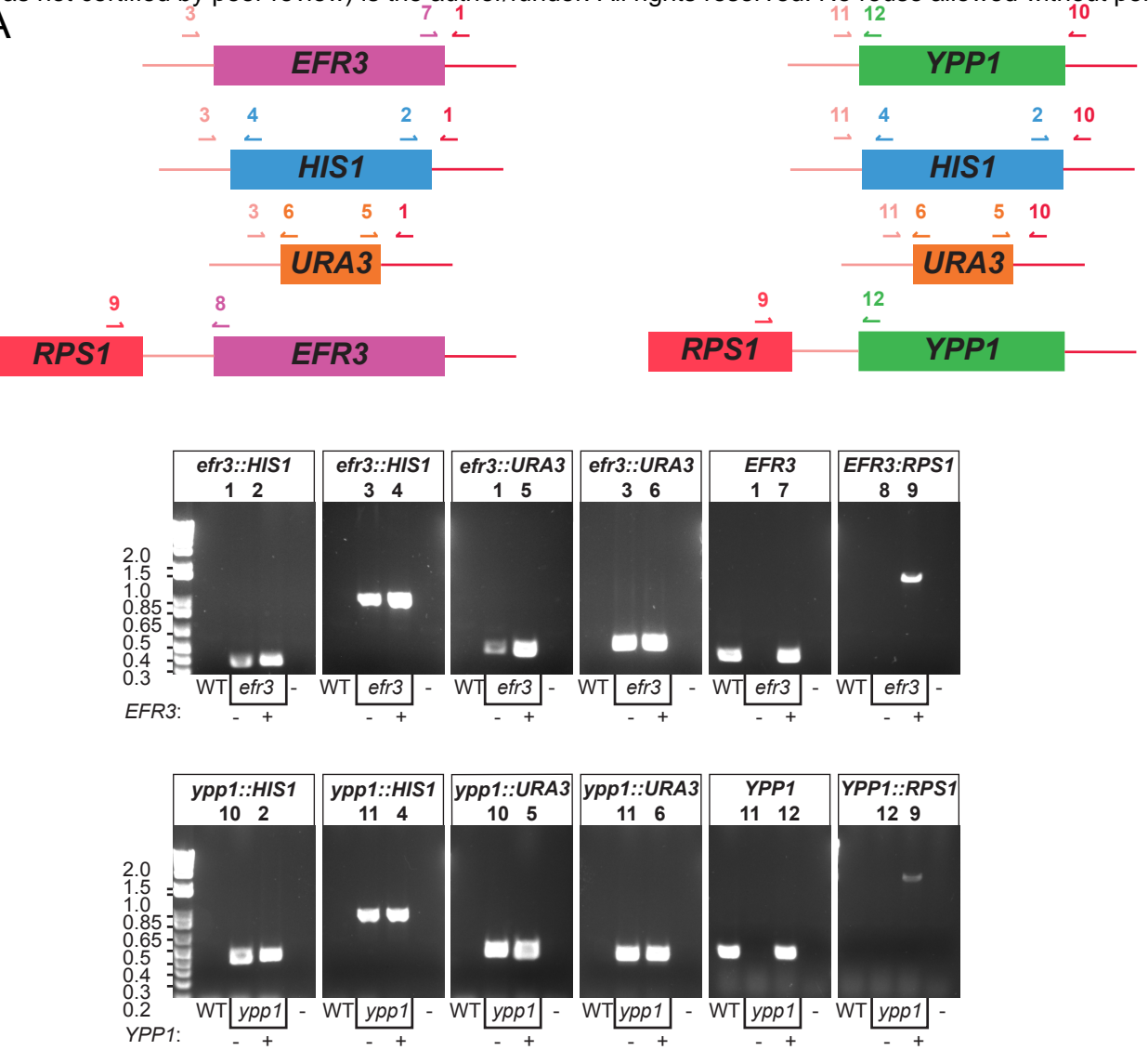

B

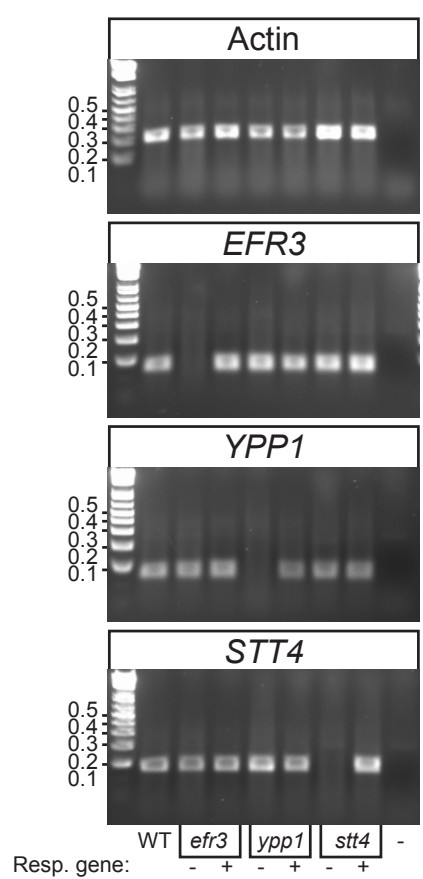

Figure S3. Molecular analyses of efr 3 and ypp1 deletion mutants. A) PCR analyses of efr 3 and ypp 1 mutants. Schematic representation of EFR3 and YPP1 with primers used for strain verification indicated (top) and PCR analyses of indicated strains (wild-type, PY4861; efr $3 \Delta / \Delta$, PY4036; efr $3 \Delta / \Delta+E F R 3$, PY4039; ypp $1 \Delta / \Delta$, PY4033; ypp $1 \Delta / \Delta+Y P P 1$, PY4040), bottom. Primers, 1 CaYpp1pup167; 2 CaHIS1pStop1008, 3 CaYpp1m3313; 4 CaHIS1mup152; 5 CaURA3p751; 6 CaURA3mup270; 7 CaYpp1m87; 8 CaRPS1p; 9 CaEfr3pup100; 10 CaEfr3m3118; 11 CaEfr3p2692; 12 CaEfr3m127. B) Efr3, yppl and stt4 mutants are only lacking the respective mRNA transcripts. RT-PCR was carried out on indicated strains (same as above and $s t t 4 \Delta / \Delta$, PY5111; stt $4 \Delta / \Delta+S T T 4$, PY5131) with primers for indicated gene amplifications. Fragments migrated at the expected sizes and ACT1 controls revealed similar amounts of cDNA in each strain. 
A

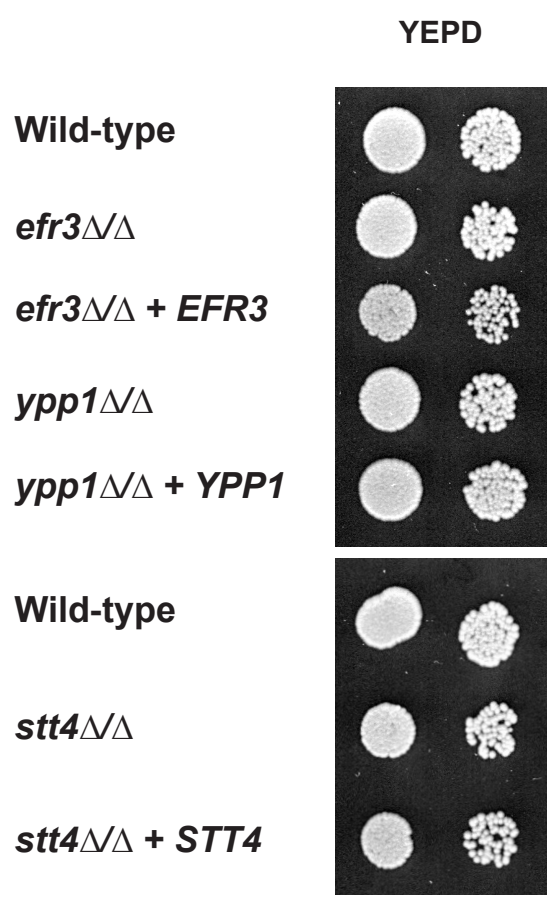

B

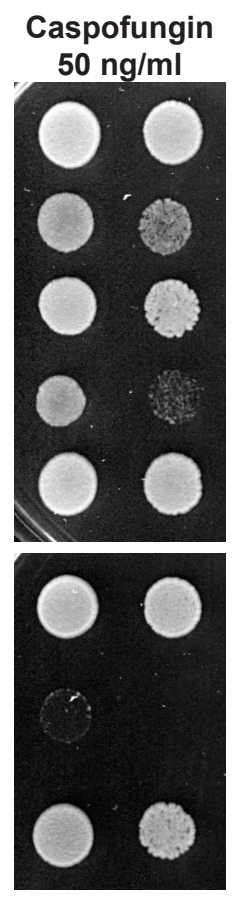

$30^{\circ} \mathrm{C}$

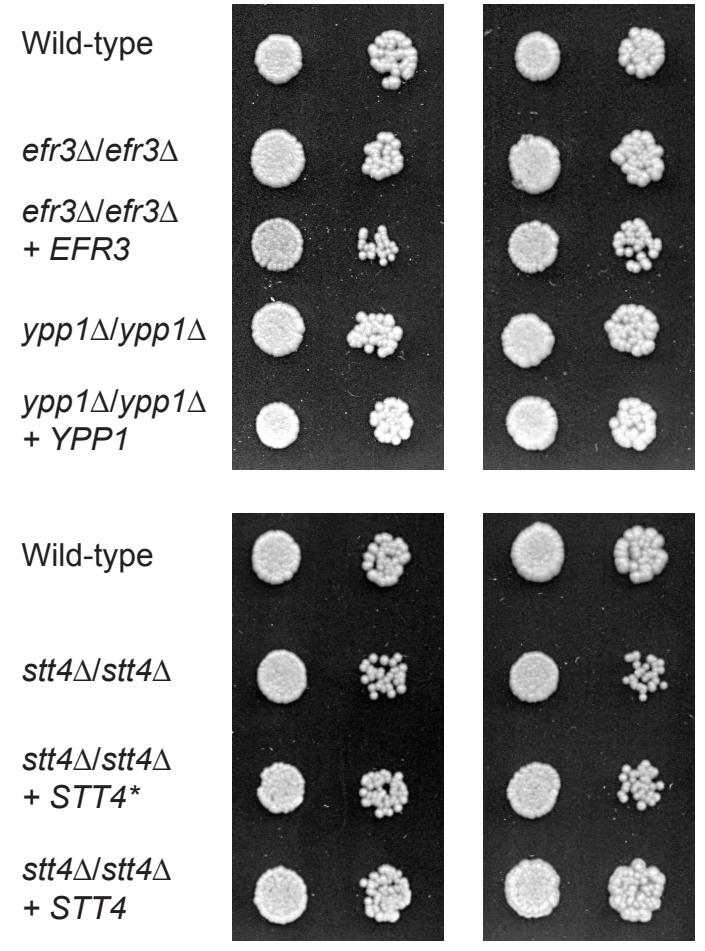

Figure S4. efr3, ypp1 and stt4 deletion mutants are increasingly sensitive to Caspofungin and grow similar to the wild-type strain at $30^{\circ} \mathrm{C}$ and $37^{\circ} \mathrm{C}$. A) Indicated strains (wild-type, PY4861; efr $3 \Delta / \Delta$, PY4036; efr $3 \Delta / \Delta+$ EFR3, PY4039; ypp $1 \Delta / \Delta$, PY4033; ypp $1 \Delta / \Delta+Y P P 1$, PY4040; stt4 $\Delta / \Delta$, PY5040; stt4 $\Delta / \Delta+S T T 4$, PY5119) were spotted on YEPD agar containing Caspofungin at the indicated concentration and incubated for 2 days at $30^{\circ} \mathrm{C}$. B) Indicated strains (wild-type, PY4861; efr $3 \Delta / \Delta$, PY4036; efr $3 \Delta / \Delta+E F R 3$, PY4039; ypp $1 \Delta / \Delta$, PY4033; ypp $1 \Delta / \Delta+Y P P 1$, PY4040; stt4 $\Delta / \Delta$, PY5040; stt4 $\Delta / \Delta+$ stt4* encoding Stt4[G1810D], PY5757; stt $4 \Delta / \Delta+S T T 4$, PY5119) were spotted on YEPD agar and incubated for 2 days at the indicated temperature. 


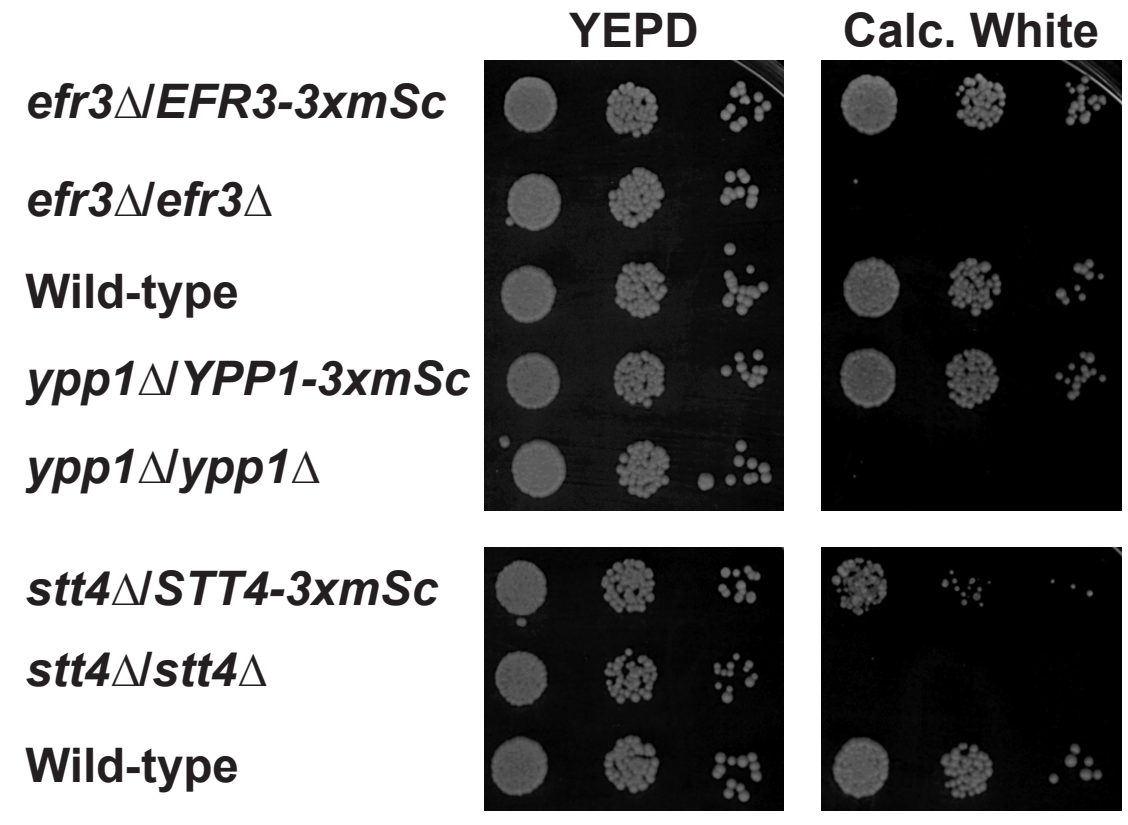

Figure S5. The Efr3, Ypp1 and Stt4 3xmScarlet fusions are functional. The indicated strains (efr3 $\Delta / E F R 3-3 x m S c$, PY5599; efr $3 \Delta / \Delta$, PY4036; wild-type, PY4861; ypp 1 $/ Y P P 1-3 x m S c$,

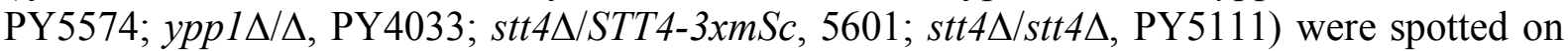
YEPD containing Calcofluor White $(25 \mu \mathrm{g} / \mathrm{mL})$ and incubated for 2 days at $30^{\circ} \mathrm{C}$. 

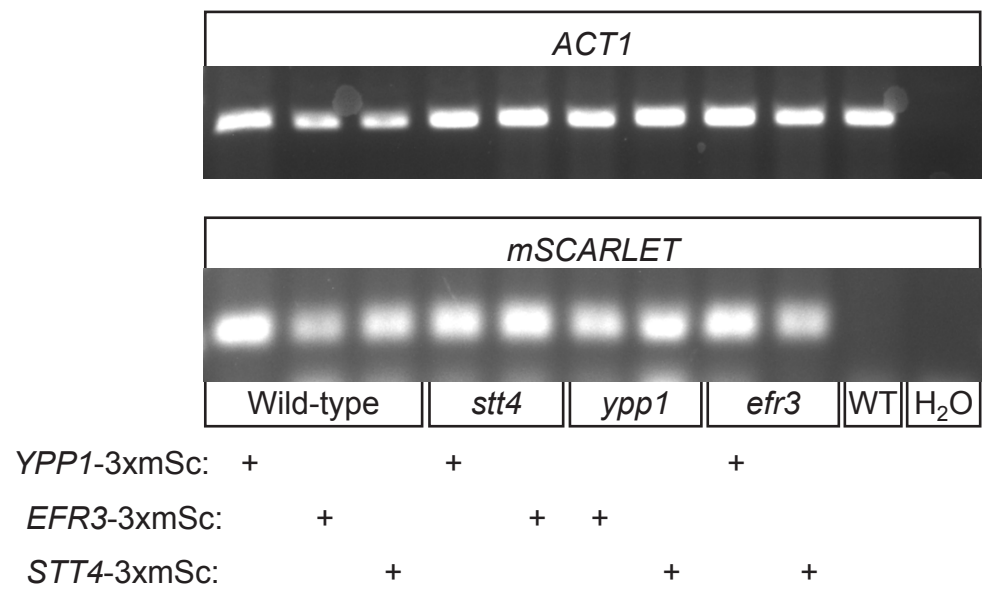

Figure S6. Transcript levels of EFR1, YPP1 and STT4 $\mathrm{mScarlet}$ fusions are not altered in the PI-4-kinase complex mutants. RT-PCR was carried out on the indicated strains (WT, PY6195 Ypp1-3xmSc, PY6197 Efr3-3xmSc, PY6193 Stt4-3xmSc; stt4 Ypp1-3xmSc, PY6134; stt4 Efr3-3xmSc, PY6140; ypp1 efr3-3xmSc, PY6138; ypp1 Stt4-3xmSc, PY6144; efr3 Ypp13xmSc, PY6136; efr3 Stt4-3xmSc; WT, PY4860) with primers (CamSCARLETp-TM, CamSCARLETm-TM, CaACT1p-TM and CaACT1m-TM) and for indicated gene amplifications. Fragments migrated at the expected sizes and ACT1 controls revealed similar amounts of cDNA in each strain. Similar results were observed with two additional mScarlet primer pairs. 

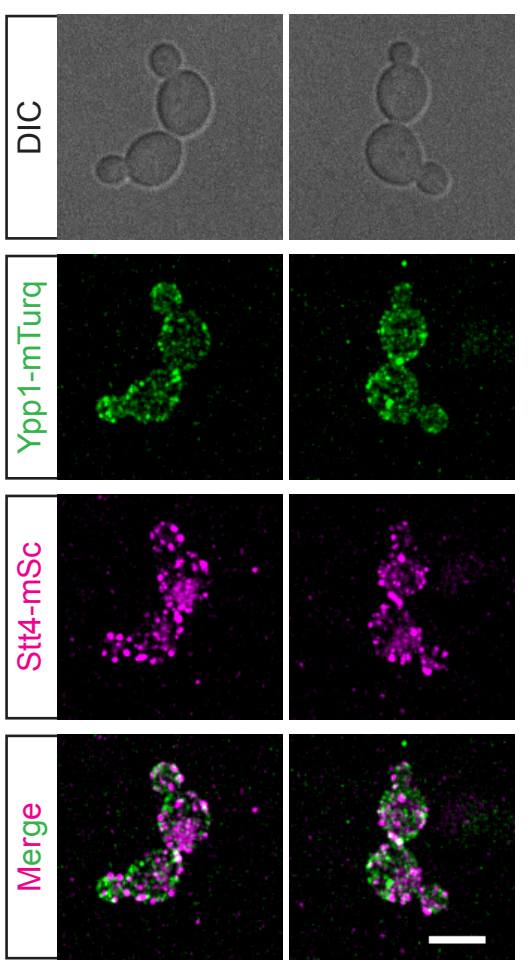

Figure S7. Ypp1 and Stt4 do not colocalize in cortical patches. A strain expressing Stt43xmScarlet (magenta) and Ypp1-mTurquoise (green), PY6201, was imaged during budding growth and maximum projections of $17 \times 0.5 \mu \mathrm{m}$ z-sections are shown. Bar is $5 \mu \mathrm{m}$. 


\section{FCS}
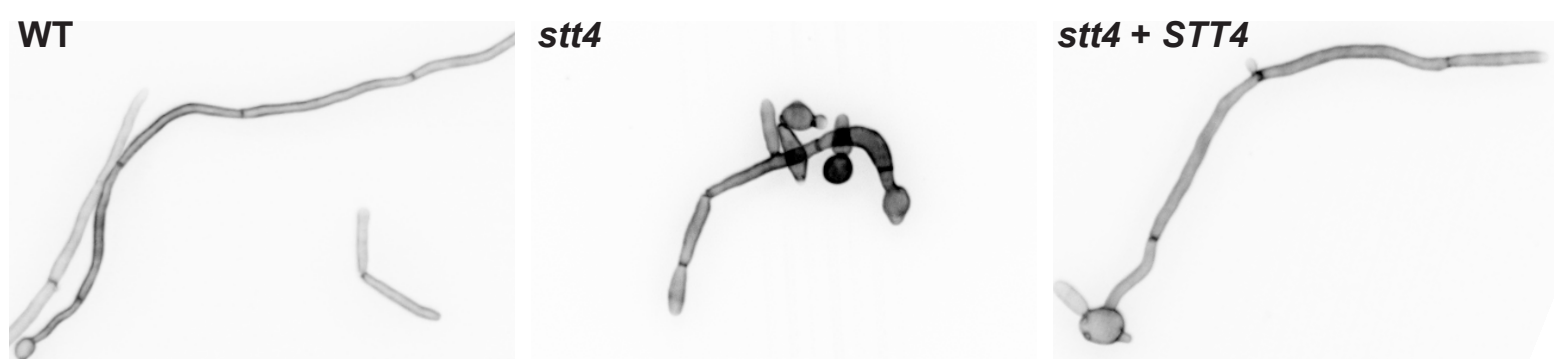

\section{Kidney homogenate}
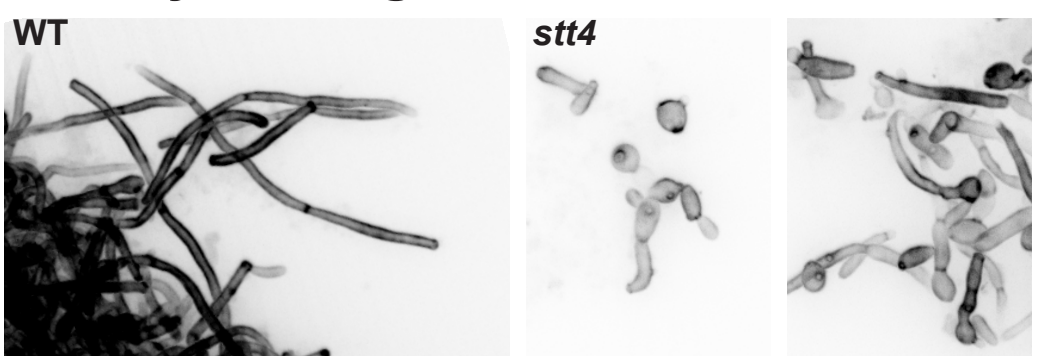

stt4 + STT4

Figure S8. The stt4 deletion mutant elongates in response to serum and murine kidney homogenates. Indicated strains (wild-type, PY4861; stt4 $/ \Delta$, PY5111; stt4 $\Delta / \Delta+S T T 4$, PY5131) were incubated with either serum or $0.4 \mathrm{~g} / \mathrm{mL}$ kidney homogenates for $6 \mathrm{Hr}$ at $37^{\circ} \mathrm{C}$, samples were fixed, stained with Calcofluor White and images (31 x $0.4 \mu \mathrm{m} \mathrm{z}$-sections) were acquired. Maximum projections are shown with an inverted LUT. Bar is $5 \mu \mathrm{m}$. 

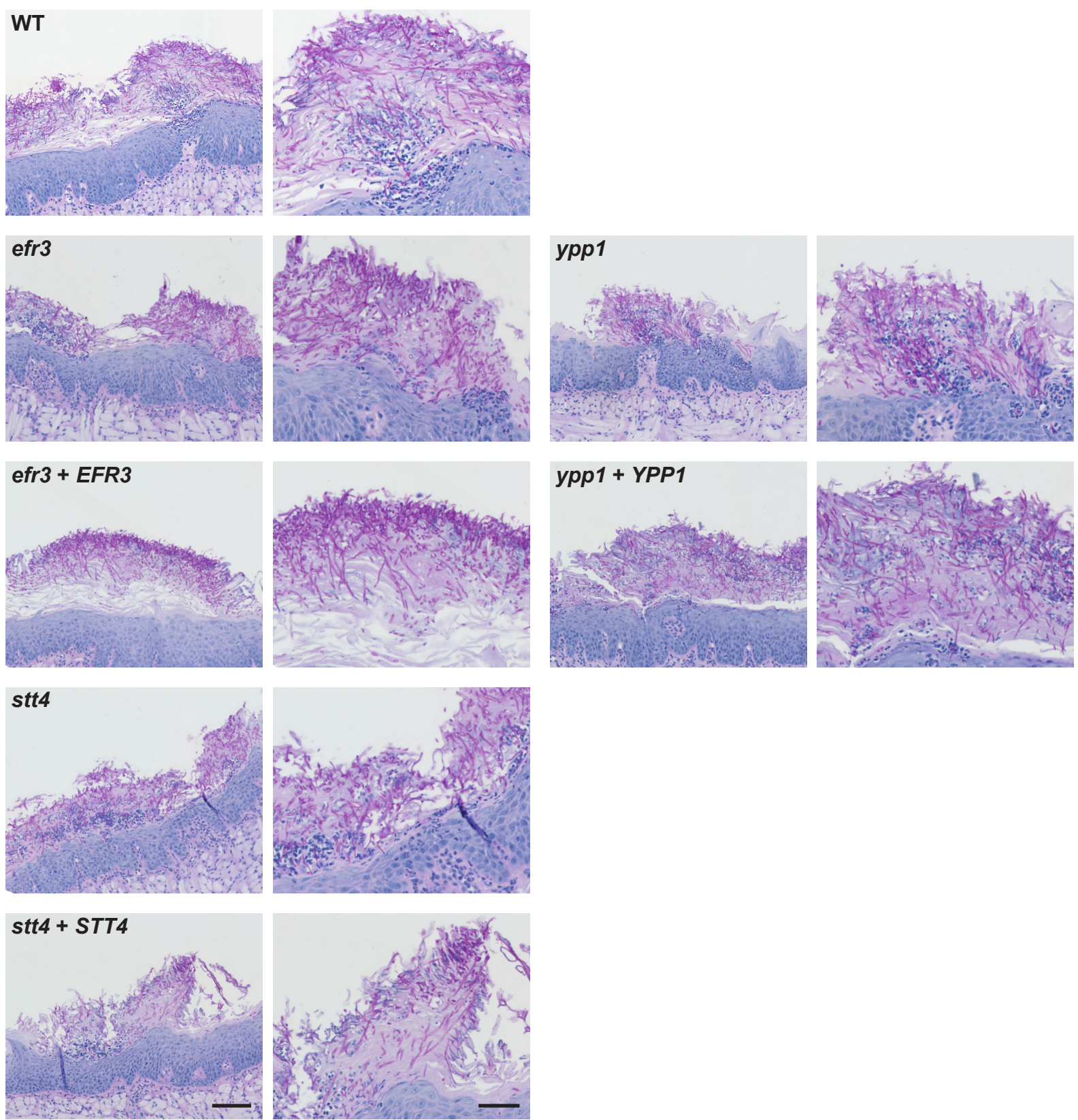

Figure S9. PI-4-kinase complex mutants can filament in a murine OPC model. Histopathology of tongue thin sections from mice infected with indicated strains (see Figure 11C). Thin sections were stained with periodic acid-Schiff stain. Images of regions of infection are shown to highlight fungal morphology, with enlargements of images on left panels (bar, $50 \mu \mathrm{m}$ ) shown on the right panels (bar, $100 \mu \mathrm{m}$ ). Note there were fewer infection sites in mice infected with the yppl mutant, which were also smaller in size. 
Table S1. Strains used in this study

\begin{tabular}{|c|c|c|}
\hline Strain & GENOTYPE & SOURCE \\
\hline BWP17 & $\begin{array}{l}\text { ura3 } \Delta:: \lambda i m m 434 / u r a 3 \Delta:: \lambda i m m 434 \text { his1 } \Delta:: \text { hisG/his1 } 1:: \text { hisG } \\
\arg 4 \Delta:: \text { hisG/arg } 4 \Delta:: \text { hisG }\end{array}$ & (1) \\
\hline PY173 & $\begin{array}{l}\text { ade } 2 \Delta: \text { :hisG/ade2 } \Delta:: \text { hisG ura3 } \Delta:: \lambda \text { imm434/ura3 } \Delta:: \lambda \text { imm434 } \\
\text { his1 } \Delta:: \text { hisG/his1 }:: \text { hisG arg } 4 \Delta:: \text { hisG/arg } 4 \Delta:: \text { hisG } \\
\text { ENO1/eno1::ENO1-tetRScHAP4AD-3xHA-ADE2 }\end{array}$ & (2) \\
\hline PY1206 & Same as BWP17 with RPS1::ARG4-pADH1-GFP-(PH Plcd $)_{2}$-GFP & (2) \\
\hline PY2578 & Same as PY173 with RPS1::ARG4-pADH1-PH ${ }^{\mathrm{FAPP} 1[\mathrm{E} 50 \mathrm{~A}, \mathrm{H} 54 \mathrm{~A}]-\mathrm{GFP}}$ & (3) \\
\hline PY2626 & $\begin{array}{l}\text { Same as PY173 with } \\
\left.\text { RPS1::ARG4-pACT1-GFP-(PH }{ }^{\mathrm{OSH} 2[\mathrm{H} 340 \mathrm{R}]}\right)_{2} \text {-GFP }\end{array}$ & (3) \\
\hline PY3239 & Same as BWP17 with RPS1::ARG4-pACT1-GFP-yeLactC2 & (4) \\
\hline PY3857 & Same as BWP17 with efr3A::HIS1/EFR3 & This study \\
\hline PY3859 & Same as BWP17 with ypp1 $:: H I S 1 / Y P P 1$ & This study \\
\hline PY3915 & Same as BWP17 with efr3 $\triangle:: H I S 1 /$ efr $3 \triangle:: U R A 3$ & This study \\
\hline PY3918 & 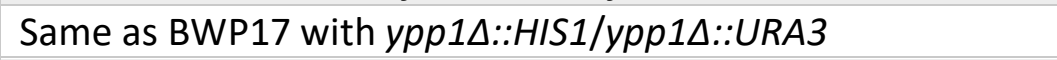 & This study \\
\hline PY3933 & $\begin{array}{l}\text { Same as PY3915 with } \\
\text { RPS1::ARG4-pADH1-PHFAP1[E50A,H54A]-GFP }\end{array}$ & This study \\
\hline PY3935 & Same as PY3915 with RPS1::ARG4-pADH1-GFP-(PH $\left.{ }^{\mathrm{Plcd}}\right)_{2}$-GFP & This study \\
\hline PY3947 & $\begin{array}{l}\text { Same as PY3915 with } \\
\left.\text { RPS1::ARG4-pACT1-GFP-(PH }{ }^{\mathrm{OSH} 2[\mathrm{H} 340 \mathrm{R}]}\right)_{2} \text {-GFP }\end{array}$ & This study \\
\hline PY3950 & $\begin{array}{l}\text { Same as PY3918 with } \\
\left.\text { RPS1::ARG4-pACT1-GFP-(PH }{ }^{\mathrm{OSH} 2[\mathrm{H} 340 \mathrm{R}]}\right)_{2} \text {-GFP }\end{array}$ & This study \\
\hline PY3951 & $\begin{array}{l}\text { Same as PY3918 with } \\
\text { RPS1::ARG4-pADH1-PHFAP1[E50A,H54A]-GFP }\end{array}$ & This study \\
\hline PY3958 & Same as PY3918 with RPS1::ARG4-pADH1-GFP-(PH $\left.{ }^{\mathrm{Plcd}}\right)_{2}$-GFP & This study \\
\hline PY4033 & Same as PY3918 with RPS1::ARG4 & This study \\
\hline PY4036 & Same as PY3915 with RPS1::ARG4 & This study \\
\hline PY4039 & Same as PY3915 with RPS1::ARG4-EFR3pEFR3 & This study \\
\hline PY4040 & Same as PY3918 with RPS1::ARG4-YPP1pYPP1 & This study \\
\hline PY4124 & Same as PY3915 with RPS1::ARG4-pACT1-GFP-yeLactC2 & This study \\
\hline PY4131 & Same as PY3918 with RPS1::ARG4-pACT1-GFP-yeLactC2 & This study \\
\hline PY4330 & Same as BWP17 with stt4D::URA3/STT4 & This study \\
\hline PY4335 & Same as BWP17 with STT4::SAT1 (stt4-DAmP)/STT4 & This study \\
\hline PY4339 & Same as PY4335 with stt4D::URA3/STT4::SAT1 (stt4-DAmP1) & This study \\
\hline PY4340 & Same as PY4335 with stt4D::URA3/STT4::SAT1 (stt4-DAmP2) & This study \\
\hline PY4341 & Same as PY4335 with stt4D::URA3/STT4::SAT1 (stt4-DAmP3) & This study \\
\hline PY4377 & Same as PY4339 with stt4D::HIS1/stt4D::URA3 & This study \\
\hline PY4378 & 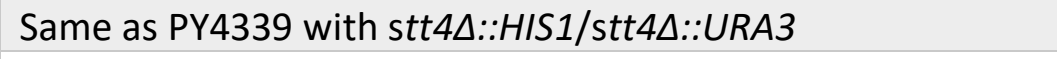 & This study \\
\hline PY4414 & Same as PY4377 with RPS1::ARG4 & This study \\
\hline PY4433 & Same as PY4377 with RPS1::ARG4-STT4pSTT4 & This study \\
\hline PY4861 & $\begin{array}{l}\text { ura3 } 1:: \lambda \text { imm434/ura3 } 4:: \lambda \text { imm } 434 \\
\text { his1::hisG/HIS1::his1::hisG } \\
\text { arg4::hisG/ARG4::URA3::arg4::hisG }\end{array}$ & $(5)$ \\
\hline PY5027 & Same as PY4377 with stt4 $\triangle:: H I S 1 /$ stt4 $\triangle:: S A T$ & This study \\
\hline PY5040 & Same as PY5027 with RPS1::URA3 & This study \\
\hline
\end{tabular}




\begin{tabular}{|c|c|c|}
\hline PY5111 & Same as PY5040 with RPS1::ARG4 & This study \\
\hline PY5119 & Same as PY5040 with stt4::STT4pSTT4-ARG4/stt4D::SAT & This study \\
\hline PY5131 & Same as PY5119 with his1::HIS1 & This study \\
\hline PY5132 & Same as PY5119 with his1::HIS1 & This study \\
\hline PY5169 & $\begin{array}{l}\text { Same as PY5040 with } \\
R P S 1:: A R G 4-p A C T 1-G F P-\left(\mathrm{PH}^{\mathrm{OSH} 2[\mathrm{H} 340 \mathrm{R}]}\right)_{2} \text {-GFP }\end{array}$ & This study \\
\hline PY5174 & Same as PY5040 with RPS1::ARG4-pACT1-GFP-yeLactC2 & This study \\
\hline PY5552 & $\begin{array}{l}\text { Same as PY5040 with } \\
\text { RPS1::ARG4-pADH1-PH }{ }^{\text {FAPP1[E50A,H54A]-GFP }}\end{array}$ & This study \\
\hline PY5554 & Same as PY5040 with RPS1::ARG4-pADH1-GFP-(PH $\left.{ }^{\mathrm{Plcd}}\right)_{2}-\mathrm{GFP}$ & This study \\
\hline PY5574 & Same as PY3859 with YPP1::3x-mSc- ARG4/ypp1A::HIS1 & This study \\
\hline PY5587 & Same as BWP17 with STT4::3x-mSc-cdHIS1/STT4 & This study \\
\hline PY5599 & Same as PY3857 with EFR3::3x-mSc-ARG4/efr3A::HIS1 & This study \\
\hline PY5601 & Same as PY4330 with STT4::3x-mSc-cdHIS1/stt4A::URA3 & This study \\
\hline PY5757 & $\begin{array}{l}\text { Same as PY5040 with } \\
\text { stt4::STT4pSTT4[G1180D]-ARG4/stt4 } \Delta:: S A T\end{array}$ & This study \\
\hline PY5808 & $\begin{array}{l}\text { Same as PY5757 with } \\
\text { stt4::STT4pSTT4[G1180D]-SAT1/stt4 }:: H I S 1\end{array}$ & This study \\
\hline PY5839 & $\begin{array}{l}\text { Same as PY5808 with } \\
R P S 1:: A R G 4-p A C T 1-G F P-\left(\mathrm{PH}^{\mathrm{OSH} 2[\mathrm{H} 340 \mathrm{R}]}\right)_{2} \text {-GFP }\end{array}$ & This study \\
\hline PY5903 & Same as PY5808 with RPS1::ARG4-pACT1-GFP-yeLactC2 & This study \\
\hline PY6134 & Same as PY5040 with YPP1::3x-mSc-ARG4 & This study \\
\hline PY6136 & Same as PY3915 with YPP1::3x-mSc-ARG4 & This study \\
\hline PY6138 & Same as PY3919 with EFR3::3x-mSc-ARG4 & This study \\
\hline PY6140 & Same as PY5040 with EFR3::3x-mSc-ARG4 & This study \\
\hline PY6142 & Same as PY3915 with STT4::3x-mSc-ARG4 & This study \\
\hline PY6144 & Same as PY3919 with STT4::3x-mSc-ARG4 & This study \\
\hline PY6193 & Same as BWP17 with STT4::3x-mSc-ARG4 & This study \\
\hline PY6195 & Same as BWP17 with YPP1::3x-mSc-ARG4 & This study \\
\hline PY6197 & Same as BWP17 with EFR3::3x-mSc-ARG4 & This study \\
\hline PY6201 & Same as PY5587 with YPP1::mTurq-ARG4 & This study \\
\hline
\end{tabular}




\section{Table S2. Plasmids used in this study}

\begin{tabular}{|c|c|}
\hline Plasmid & Source \\
\hline pExpARG-pADH1-GFP-(PH ${ }^{\mathrm{OSH}}\left[\mathrm{H}_{340 \mathrm{R}]}\right)_{2}$-GFP & (3) \\
\hline pExpARG-pADH1-PH FAPP1[E50A, H54A]_GFP & (3) \\
\hline pExpARG & (3) \\
\hline pExpARG-pACT1-GFP-yeLactC2 & (4) \\
\hline pExpARG-pADH1-GFP-PH ${ }^{\mathrm{Plc} \delta}-\mathrm{PH} \mathrm{H}^{\mathrm{Plc} \delta}$-GFP & $(2)$ \\
\hline pGEM-HIS1 & (1) \\
\hline pGEM-URA3 & (1) \\
\hline pFA-SAT1 & (6) \\
\hline pFA-HIS1 & (6) \\
\hline pFA-URA3 & (6) \\
\hline pFA-ARG4 & (6) \\
\hline pFA-3x-mSc-ARG4 & (7) \\
\hline pFA-3x-mSc-cdHIS1 & $\begin{array}{l}\text { (C. Puerner, M. Bassilana, } \\
\text { and R. A. Arkowitz, in } \\
\text { preparation) }\end{array}$ \\
\hline $\mathrm{p} \operatorname{Exp} A R G 4-\mathrm{p} E F R 3-E F R 3$ & This study \\
\hline pExpARG4-pYPP1-YPP1 & This study \\
\hline pExpARG4-pSTT4-STT4 & (2) \\
\hline pExpARG4-pSTT4-STT4-STT4t::ARG4 & This study \\
\hline pExpARG4-pSTT4-STT4*-STT4t::ARG4 & This study \\
\hline pFA-GFPY-NAT & (8) \\
\hline pFA-mTurq-ARG4 & $\begin{array}{l}\text { (C. Puerner, M. Bassilana, } \\
\text { and R. A. Arkowitz, in } \\
\text { preparation) }\end{array}$ \\
\hline
\end{tabular}




\section{Table S3. Primers used in this study}

\begin{tabular}{|c|c|}
\hline Primer & Sequence $5^{\prime} \rightarrow 3^{\prime}$ \\
\hline CaEfr3mKO & $\begin{array}{l}\text { ccttttacctagaaggtattttgacttgggtatctttgca } \\
\text { actgtctctctctcgctttagcttaaacaacaaacgcagcT } \\
\text { TTCCCAGTCACGACGTT }\end{array}$ \\
\hline CaEfr3pKO & $\begin{array}{l}\text { ctttctattggttaattaaatcctgttttgattcaatagt } \\
\text { ttcttcaacttccagatccaagaatgaatttgtttcaacat } \\
\text { TGTGGAATTGTGAGCGGATA }\end{array}$ \\
\hline CaEfr3mXFPS2 & $\begin{array}{l}\text { ggataaaaaatgcagtaatgttccttttacctagaaag } \\
\text { gtattttgacttgggtatctttgcaactgtctctctctcgc } \\
\text { tttagcTCTGATATCATCGATGAATTCGAG }\end{array}$ \\
\hline CaEfr3pXFPS1 & $\begin{array}{l}\text { gtgttgaataaaatatgcaaactacagatgttgattcaat } \\
\text { tttaagtggtcttgaaagtgaagacgaagctgcgtttgttg } \\
\text { ttGGTGCTGGCGCAGGTGCTTC }\end{array}$ \\
\hline CaEfr3m127 & СTCTACGAGTGGATGCATA \\
\hline CaEfr3m3118 & ССТСАААСТТАСАСАСТСТТСС \\
\hline CaEfr3m3183Notl & TATAGCGGCCGCctgcaaataggggactcttaac \\
\hline CaEfr3p595 & GCAAACAACCCACAAAGCA \\
\hline CaEfr3p1304 & CGTCCACTGGAGAACAAAT \\
\hline CaEfr3p1980 & GGATGAGCAATATAGTCAT \\
\hline CaEfr3p2593 & CGAACTGTCGATAGTGTT \\
\hline CaEfr3p2692 & ATGTCAACACATAGGGGTA \\
\hline CaEfr3pup100 & TTCATCAAGAATATCCTT \\
\hline CaEfr3pup960Xhol & GCCTCGAGgccccactcccactcccccaac \\
\hline CaStt4mKO & $\begin{array}{l}\text { ctatagataacatacactattaacgcctccactagtaagg } \\
\text { aataccattggtaagtctttggaattcatTTTCCCAGTCAC } \\
\text { GACGTT }\end{array}$ \\
\hline CaStt4pKO & $\begin{array}{l}\text { cagaaagaacagaaagctatggattattctgggattaccC } \\
\text { gtggctcaattcgtgctgaagcccttaagTGTGGAATTGTG } \\
\text { AGCGGATA }\end{array}$ \\
\hline CaStt4mXFPS2 & $\begin{array}{l}\text { GGTTGCAGCCAAAAACACGAGCGGATAACAAAGTCCAATGT } \\
\text { CAATAATTACTATAGATAAACATACACTATTAACGCСTCCA } \\
\text { TCTGATATCATCGATGAATTCGAG }\end{array}$ \\
\hline CaStt4pXFPS1 & $\begin{array}{l}\text { CTTTAGAAAATTGATCAAGAAATCGATGGAAAGTTTCTACA } \\
\text { CTAAAGGTTATGATGAATTCCAAAGACTTACCAATGGTATT } \\
\text { CCTTACGGTGCTGGCGCAGGTGCTTC }\end{array}$ \\
\hline CaStt4G1810DmEcoRV & $\begin{array}{l}\text { GGTGCAGCCTCAAАCCTAACCCCATCAGGAACGATATCAAA } \\
\text { ACAGAACССАААATCAАTATG }\end{array}$ \\
\hline CaStt4G1810DpEcoRV & $\begin{array}{l}\text { CATATTGATTTTGGGTTCTGTTTTGATATCGTTCCTGATGG } \\
\text { GGTTAGGTTTGAGGCTGCACC }\end{array}$ \\
\hline CaStt4m160 & GTAATTTGGGAGTGGCCG \\
\hline CaStt4m4255 & CTGATAGCAGGTAATGGGTC \\
\hline CaStt4m5056 & САTGGAAATCAATTGTAAGGC \\
\hline CaStt4m5130 & GGAGCAGTTGCAGTAACT \\
\hline CaStt4m5543 & ССТТТСАСАСАСААСТСТТС \\
\hline CaStt4m5857 & ttgtaacaaaggttgcagcc \\
\hline CaStt4m6102 & ССTTGTGGTTGCTATTTGG \\
\hline CaStt4m6286 & GCTGGTCTTGGGGGGTTGG \\
\hline CaStt4m6784 & АTTTСАТАTССАGСААTTATGCC \\
\hline CaStt4m6924 & ССАСТTСАТTAААСАAGCC \\
\hline
\end{tabular}




\begin{tabular}{|c|c|}
\hline CaStt4m4115KI & $\begin{array}{l}\text { CTAGTATCTGAAGGATTCATTGAATGTAACCACACAGTAAA } \\
\text { TTTACAAATCTCATCATCCATAAAATATAAAAGAATTTTCa } \\
\text { tagcttttctgttctttctg }\end{array}$ \\
\hline CaStt4m4864KI & $\begin{array}{l}\text { GATTTTAAATGTCGCCATAAATGGTGCCTTGGCATGTGATT } \\
\text { GTAATGGTCTACCCAGTTTACGATTAATATCAATAACAACC } \\
\text { atagcttttctgttctttctg }\end{array}$ \\
\hline CaStt4m6600Notl & TCTGCGGCCGCcgtggatcacaacattgtg \\
\hline CaStt4mupSacll & TTACCgCggCATAGCTTTTCTGTTCTTTCTGTTTTCTTG \\
\hline CaStt4p4100 & CATTGCGTACCGATGAAG \\
\hline CaStt4p4884 & GCСАTCCAATCCTGATGGGGTTG \\
\hline CaStt4p5199 & GGGAAGAGAAGCTGTTAATGG \\
\hline CaStt4p5439 & GTTCCTGGTGGGGTTAGG \\
\hline CaStt4p5488 & GGAGGTAATGATCAAACTC \\
\hline CaStt4p5519 & GGTTTGAAGAGTTGTGTGTG \\
\hline CaStt4p5740 & GGTTATGATGAATTCCAAAG \\
\hline CaStt4p5857 & ttgtaacaaaggttgcagcc \\
\hline CaStt4p5974 & CGGTCAATAATCTTCGGGATTAAAG \\
\hline CaStt4pup168 & CCСАCCACCAACGCAATTATC \\
\hline CaStt4pup325 & caatcacgtgcattctccac \\
\hline CaStt4pup617S1 & $\begin{array}{l}\text { GAGACACTTTGATTGAAACTTATTCTTAAATTGGGTTTCTC } \\
\text { AAAATATAGGAATTAAAAAGGGGTTTATGTATAGAGTGTTA } \\
\text { Ggaagcttcgtacgctgcaggtc }\end{array}$ \\
\hline CaStt4pupSpel & GGCactagtCCCGCTCCCAACTAATCAAC \\
\hline CaStt4term_mAsclPmel & $\begin{array}{l}\text { agaatagaggaagaaaaaaaagggTTTaaaccatGgcGCG } \\
\text { cCagatctcaacgatacttgtacg }\end{array}$ \\
\hline CaStt4term_pAsclPmel & $\begin{array}{l}\text { cgtacaagtatcgttgagatctGGCGCgcCatggtttAAAc } \\
\text { ccttttttttcttcctctattct }\end{array}$ \\
\hline CaYpp1mKO & $\begin{array}{l}\text { attgattttttatatattctccttttttgatagtctgtttc } \\
\text { gctctttgtctattaaagttcatcaaatataaattcCCTTT } \\
\text { CCCAGTCACGACGTT }\end{array}$ \\
\hline CaYpp1pKO & $\begin{array}{l}\text { cacatcctattaagttgatcttttattttggcttttaatta } \\
\text { ttttaattcCcaacattatagatgtatattgagagttcgTG } \\
\text { TGGAATTGTGAGCGGATA }\end{array}$ \\
\hline CaYpp1mXFPS2 & $\begin{array}{l}\text { gctttctgtacatattttattgattttttatatattctcct } \\
\text { tttttgatagtctgtttcgctctttgtctaTCTGATATCAT } \\
\text { CGATGAATTCGAG }\end{array}$ \\
\hline CaYpp1pXFPS1 & $\begin{array}{l}\text { gataaatattattgacgaaagtttatggagatgtattga } \\
\text { attagaagataaacgaccagttagggaatttatatttgatg } \\
\text { aacttGGTGCTGGCGCAGGTGCTTC }\end{array}$ \\
\hline CaYpp1m87 & TTGTTGGAATTCCAATAC \\
\hline CaYpp1m3313 & АСTTACAAGGCTCATATC \\
\hline CaYpp1m3577Notl & TATAGCGGCCGCcaaacgaggaacaatcaaagtgtcg \\
\hline CaYpp1p503 & GTGCGGGGAACAATGGAGT \\
\hline CaYpp1p1173 & CAACCCATTGAAAAGTTTC \\
\hline CaYpp1p1929 & GAAGCATCGTCAACGCAAT \\
\hline CaYpp1p2637 & TCCACGTCATCAACATGAT \\
\hline CaYpp1p3073 & GGATATAATAATCCAGAA \\
\hline CaYpp1pup167 & CAGTTTAGCCGGTTGTCC \\
\hline CaYpp1pup962Xhol & GCCTCGAGgtcacattaactcctatacttgc \\
\hline
\end{tabular}


CaARG4ExchS1

CaARG4ExchS2

CaARG4Ascl-S1

CaARG4Pmel-S2

CaARG4m537

CaHIS1mup152

CaHIS1m836

CaHIS1pStop1008

CaHis1p214

CaURA3mup270

CaURA3m81

CaURA3mSAT1

CaURA3p751

CaURA3pSAT1

CaURA3pXhol

CaRPS1p

CaSATDAmPmKO

CaSATDAmPmS2

CaSATDAmPpS1

CaSATm596

CaSATm1638

CaSATp1315

CaSATp1734

CaSATp1315

CaSATp1734

CaGFPm106

CamScarletm108

CamTurq2m419

CaACT1m-TM

CaACT1p-TM

CaALS3p-TM

CaALS3m-TM

CaECE1p-TM

CaECE1m-TM

CaEFR3m-TM

CaEFR3p-TM atgtcacaacaacaagataaacaacctagtgaaataaatt atggggtggtcgtttcaccggtgccactgatccattgatgg GAAGCTTCGTACGCTGCAGGTC

ttaacttaagattgattttaaattttctaattgttttaaaa cggcacttttagcagtaccaccaagagcatctcttctttcc

tctggcgcgcCAAGCTTCGTACGCTGCAGGTC

agggtttaaacTCTGATATCATCGATGAATTCGAG

GTGCCCATCTAATAGGTTGAG

CGAAATGGCCTCCCCTAC

tcgtccattacattaccg

CTTTAGATGGATCGACATTGG

gacttgggtataactggg

ggcactacagcaactttc

CATAAATTGGTTTTCTTCAGTTCC

tataattggccagtttttttcaaataagcattccaaccagc atctctatacctttaccttcaatatctggatcCGTTAGTA TCGAATCGACAGC

GAAGGTAAAAGGTATAGAAATGC

atgacagtcaacactaagacctatagtgagagagcagaaac tcatgcctcaccagtagcacaacgattatttcgattaGATA TCAAGCTTGCCTCGTC

gttgctgtagtgccattgaCtcgAGacactaattctgtatt agtcattcc

GAGGATTGAGCGTAAGTAG

cgatgaattcgagctccaccgcggtggcggccgctctagaa ctagtggatcccccgggctgcaggaccacctttgattgTTT CCCAGTCACGACGTT

CGATGAATTCGAGCTCCACCGCGGTGGCGGCCGCTCTAGAA CTAGTGGATCCCCCGGGCTGCAGGACCACCTTTGATTGtct gatatcatcgatgaattcgag

CAAGAAATCGATGGAAAGTTTCTACACTAAAGGTTATGATG AАTTCCAAAGACTTACCAATGGTATTCCTTACTAgaagctt cgtacgctgcaggtc GTTTTCCCACCСТАСССAT AATGCCGCCGAGAGTAAAG TCTACCAGAAGTGTGAGCC gtgaagtgtgaagggggaga TCTACCAGAAGTGTGAGCC gtgaagtgtgaagggggaga ccggagacagaaatttg CATGACCATTCATTGAACCTTCC GACCTAAAATATTACСАTСTTC ACATTTGTGGTGAACAATGG ATGTTCCCAGGTATTGCTGA TTGGCAACGTGCACCTTTC GTGGTCACGGCGGTAGTACTG CCAGAAATTGTTGCTCGTGTTG CAGGACGCCATCAAAAACG TCTTCCCATCATCTTCAACGT ACAACCCACAAAGCAGCATA 


\begin{tabular}{|l|l|}
\hline CaHGC1p-TM & AAAGCTGTGATTAAATCGGTTTTGA \\
\hline CaHGC1m-TM & AATTGAGGACCTTTTGAATGGAAA \\
\hline CaHWP1p-TM & CGGAATCTAGTGCTGTCGTCTCT \\
\hline CaHWP1m-TM & TAGGAGCGACACTTGAGTAATTGG \\
\hline CaMSS4m-TM & AATCCCTCCATCATGACCATAAA \\
\hline CaMSS4p-TM & AAATCTTTTGATAAACGTGCCCTTA \\
\hline CamSCARLETm-TM & TTTTTGCATAACTGGACCATCTG \\
\hline CamSCARLETp-TM & GTGGTGCTGTTACTGTTACTCAAGATAC \\
\hline CaPIK1 $\alpha$-TM & GCTGCGATTCTCATCTGATCAGC \\
\hline CaPIK1 $\alpha$-TM & CCCCTTCTATTACGGAGCAACTG \\
\hline CaSAC1m-TM & TTCAACACCACCTCCCAGTGT \\
\hline CaSAC1p-TM & CCACAGCAACAGATGGAACTCA \\
\hline CaSAP4p-TM & TCCCTTTTCCTTAACTCTCCTGAA \\
\hline CaSAP4m-TM & AGCCGCTATACTTGGCCTTGT \\
\hline CaSAP5p-TM & CAATTATTTGCTAACGTTTGGTCTACTAG \\
\hline CaSAP5m-TM & CTTCGCCGCTTTGAAAACC \\
\hline CaSAP6p-TM & TCTCCAGGGTTTGTTACCTTAGACTT \\
\hline CaSAP6m-TM & TTAGATTCGGCAGTTGGATCATC \\
\hline CaSTT4m-TM1 & AGCCCATCAACACCATATTTTTCT \\
\hline CaSTT4p-TM1 & CCATTGATTTTGAAAGGGTTAAGTT \\
\hline CaSTT4m-TM2 & CGGTACGCAATGGTAATTATTCAT \\
\hline CaSTT4p-TM2 & GATCGTGAATGGTCCGCAAT \\
\hline CaTDH3p-TM & ATCCCACAAGGACTGGAGA \\
\hline CaTDH3m-TM & GCAGAAGCTTTAGCAACGTG \\
\hline CaYPP1m-TM & TGGTGTGGGTTGTTCTTTTTC \\
\hline CaYPP1p-TM & AAATGGTCCCATCGTCCAA \\
\hline & \\
\hline
\end{tabular}




\section{References}

1. Wilson RB, Davis D, Mitchell AP. 1999. Rapid hypothesis testing with Candida albicans through gene disruption with short homology regions. J Bacteriol 181:1868-74.

2. Vernay A, Schaub S, Guillas I, Bassilana M, Arkowitz RA. 2012. A steep phosphoinositide bis-phosphate gradient forms during fungal filamentous growth. J Cell Biol 198:711-30.

3. Ghugtyal V, Garcia-Rodas R, Seminara A, Schaub S, Bassilana M, Arkowitz RA. 2015. Phosphatidylinositol-4-phosphate-dependent membrane traffic is critical for fungal filamentous growth. Proc Natl Acad Sci U S A 112:8644-9.

4. Labbaoui H, Bogliolo S, Ghugtyal V, Solis NV, Filler SG, Arkowitz RA, Bassilana M. 2017. Role of Arf GTPases in fungal morphogenesis and virulence. PLoS Pathog 13:e1006205.

5. Bassilana M, Blyth J, Arkowitz RA. 2003. Cdc24, the GDP-GTP exchange factor for Cdc42, is required for invasive hyphal growth of Candida albicans. Eukaryot Cell 2:9-18.

6. Schaub Y, Dunkler A, Walther A, Wendland J. 2006. New pFA-cassettes for PCR-based gene manipulation in Candida albicans. J Basic Microbiol 46:416-29.

7. Silva PM, Puerner C, Seminara A, Bassilana M, Arkowitz RA. 2019. Secretory Vesicle Clustering in Fungal Filamentous Cells Does Not Require Directional Growth. Cell Rep 28:2231-2245 e5.

8. Milne SW, Cheetham J, Lloyd D, Aves S, Bates S. 2011. Cassettes for PCR-mediated gene tagging in Candida albicans utilizing nourseothricin resistance. Yeast 28:833-41. 